\title{
]jfis
}

Received: May 28, 2019

Revised : Dec. 8, 2020

Accepted: Dec. 10, 2020

Correspondence to: Jiří Močkoř

(Jiri.Mockor@osu.cz)

(T) Khe Korean Institute of Intelligent Systems

(c) This is an Open Access article distributed under the terms of the Creative Commons Attribution Non-Commercial License (http://creativecommons.org/licenses/ by-nc/3.0// which permits unrestricted noncommercial use, distribution, and reproduction in any medium, provided the original work is properly cited.

\section{Powerset Theory of Fuzzy Soft Sets}

Jiří Močkoř

Institute for Research and Applications of Fuzzy Modeling, University of Ostrava, Ostrava, Czech Republic

\begin{abstract}
Fuzzy powerset theory is defined by a monad, and therefore it can be applied in computer science. Fuzzy soft sets generalize fuzzy sets and have considerable application potential in, for instance, decision-making and optimization. In this study, we prove that fuzzy soft sets also give rise to a powerset theory, which is also defined by a monad. As in the case of fuzzy sets, in fuzzy soft set theory, it is possible to use several theoretical constructions requiring the existence of a powerset theory and monads. We describe the construction of fuzzy soft relations as an example of the use of monads in fuzzy soft sets.
\end{abstract}

Keywords: Fuzzy soft set, Monad powerset theory, Fuzzy soft relations

\section{Introduction}

Several methods have been developed for problems related to incomplete or inaccurate information. Examples of such methods are, for example, the theory of probability, fuzzy set theory, interval mathematics, and rough set theory. These can be considered mathematical methods for handling imperfect knowledge, and each have their own theoretical foundation and range of potential applications. However, it should be noted that even though the last three theories are quite different, they share several common features, and under certain conditions, we can transform theoretical results of one theory into results of the other. This process then leads to the emergence of new theories at the borders of these basic theories. This enables the use of diverse theoretical tools, thus increasing their application potential.

An example of such a boundary theory that does not fully fit in these basic areas is soft set theory. This is a rather recent approach for modeling uncertainty and was introduced by Molodtsov [1] in 1999, who established the fundamental results and proposed possible applications. Further extensions of the theoretical part and applications of soft sets subsequently appeared, for example, Maji et al. [2, 3], Majumdar and Samanta [4], Aktas and Cagman [5], Feng et al. [6], Chen et al. [7], and Mushrif et al. [8]. Both fuzzy set theory and soft set theory are concerned with problems that involve vagueness and uncertainties from different areas. Hence, the combination of these two approaches naturally led to the concept of fuzzy soft sets as a fuzzy generalization of soft sets. This was introduced by Maji et al. [9], where the basic properties of fuzzy soft sets were provided. Several studies have since appeared, extending the theoretical features of fuzzy soft sets (e.g., [10-13]).

Recently, the properties and applications of soft and fuzzy soft sets have attracted considerable attention. Xiao et al. [13] studied a synthetic evaluation method for business competitive capacity. Zou and Xiao [14] exploited the link between soft sets and data analysis in incomplete information systems. Pei and Miao [15] demonstrated that soft sets are a class of 
special information systems. Mushrif et al. [8] presented a new algorithm based on soft set theory for classification of natural textures. Kovkov et al. [16] considered optimization problems in the framework of soft set theory. Zou and Xiao [14] presented data analysis approaches for soft sets under incomplete information. Finally, Majumdar and Samanta [4] studied similarity measures for soft sets.

From this review, it follows that fuzzy soft set theory is a highly lively research area with great application potential. However, fuzzy soft set theory itself and its position in uncertainty theories have not been sufficiently investigated. Only a limited number of studies are concerned with the theoretical properties of fuzzy soft sets and their relation to, for example, classical fuzzy sets, or general categorical properties of fuzzy soft sets. Fuzzy set theory already has a large system of theoretical techniques that allow combining fuzzy sets with, for example, standard algebraic and topological methods; however, in the case of fuzzy soft sets, such techniques have not been systematically developed.

In fuzzy set theory, one of the key theoretical techniques that allows the application of standard algebraic and topological methods to fuzzy sets is powerset theory. Powerset theories are widely used in algebra, logic, topology, and computer science. The standard example of a powerset theory is based on the powerset object $P(X)=\{A: A \subseteq X\}$ of a set $X$, and the corresponding (Zadeh's) extension of a mapping $f: X \rightarrow Y$ to the map $f_{P}: P(X) \rightarrow P(Y)$ is widely used in almost all branches of mathematics and their applications, including computer science. As classical set theory can be considered a special case of fuzzy set theory, powerset objects associated with fuzzy sets were soon investigated as generalizations of classical powerset objects. This was first carried out by Zadeh [17], who defined $[0,1]^{X}$ (or $L^{X}$, where $L$ is an appropriate ordered structure) as a new powerset object $Z(X)$ instead of $P(X)$, and introduced the new powerset operator $f_{Z}: Z(X) \rightarrow Z(Y)$. Zadeh's extension was extensively studied by Rodabaugh [18] for lattice-valued fuzzy sets. in [18], the author provided a serious basis for further research on powerset objects and operators. This new approach to powerset theories was based on the application of the theory of monads in clone form, introduced by Manes [19]. A special example of monads in clone form was introduced by Rodabaugh [20] as a special structure describing powerset objects.

Instead of a monad in clone form, a more explicit powerset theory was introduced by Rodabaugh [20] as a structure describing powerset objects. A slight modification of this structure defined in a category $\mathbf{K}$ is represented by a system $\mathbf{T}=(T, \rightarrow, V, \eta)$, where $T: \mathbf{K} \rightarrow C S L A T$ is an object function representing a powerset generator (where CSLAT is the category of complete $\bigvee$-semilattices), $\rightarrow$ is a forward powerset operator such that for each $f: X \rightarrow Y$ in $\mathbf{K}$, $f_{T}: T(X) \rightarrow T(Y)$ in $C S L A T, V: \mathbf{K} \rightarrow$ Set is a concrete functor, and for each object $X, \eta_{X}: V(X) \rightarrow T(X)$ is a map in the category Set of sets. He then proved that some powerset theories can be generated by algebraic theories. In particular, $T(X)$ can be considered to consist of objects of a category $\mathbf{K}$ rather than of semilattices only.

Unfortunately, such a powerset theory is not available for fuzzy soft sets. The existence of such a theory would allow not only the application of standard algebraic and topological techniques to this area, but also the precise definition of the relationships between classical fuzzy sets and fuzzy soft sets.

Accordingly, this study focuses on obtaining a fuzzy soft set powerset theory. Our objective is not only to provide an analog of classical powerset theory for fuzzy soft sets, as in the case of classical fuzzy sets, but also to handle the case of $L$-valued fuzzy soft sets for different lattices $L$. Hence, instead of the so-called fixed-basis fuzzy soft set powerset theory, where the value lattice $L$ is fixed, we consider the so-called variable-basis fuzzy soft set powerset theory, where the value lattices can be different. To this end, we first prove that, as in the case of fixed-basis fuzzy set powerset theory, a variable-basis fuzzy set powerset theory exists and is defined by a suitable monad. Subsequently, we show that there exists a variable-basis fuzzy soft set powerset theory, which is a generalization of fuzzy set powerset theory, and we prove that it is also defined by a suitable monad. This allows us to use a series of constructs from fuzzy set theory that, by their very nature, require the existence of a powerset theory defined by a monad. To illustrate this, we use fuzzy soft set powerset theory and monads to define the theory of fuzzy soft relations and the composition of these relations.

All these results prove that fuzzy set powerset theory is a special case of fuzzy soft set powerset theory. However, we also prove that fuzzy soft set powerset theory can be understood as a special case of fuzzy set powerset theory, as instead of fixedbasis theories, we consider a variable-basis theory. Thereby, we obtain a detailed description of the relationships between the two theories. 


\section{Preliminaries}

Let $U$ be an initial universe, and $X$ be the set of all possible parameters, which are properties of objects in $U$. An $L$-valued fuzzy set in $U$ is a mapping $s: U \rightarrow L$, where $L$ is a complete lattice, the largest and smallest elements of which are $1_{L}$ and $0_{L}$, respectively. The category of complete lattices with complete lattice homomorphisms is denoted by $\mathcal{C}$. For $L \in \mathcal{C}$, the set of all $L$-valued fuzzy sets in $U$ is denoted by $L^{X}$. Instead of a lattice $L$, we occasionally consider a lattice $L$ with a tnorm (denoted by $\otimes$ ) such that $a \otimes \bigvee_{i \in I} a_{i}=\bigvee_{i \in I}\left(a \otimes a_{i}\right)$. An example of a complete lattice with a t-norm is a complete residuated lattice (see, e.g., [21]).

We also use the category $C S L A T$ of complete $\bigvee$-semilatices with complete $\bigvee$-preserving homomorphisms. As $S \in C S L A T$ is a complete semilattice, it is also a complete lattice. The only difference between the category of complete lattices $\mathcal{C}$ and the category of complete $\bigvee$-semilattices $C S L A T$ is that the morphisms in the former preserve both complete suprema and infima, whereas in the latter, we require that the morphisms preserve only complete suprema.

We use the following definition of a fuzzy soft set, introduced in [9].

Definition 1. A pair $(E, s)$ is called a fuzzy soft set in a space $(X, U, L)$ if $E \subseteq X$ and $s: E \rightarrow L^{U}$.

The interpretation of individual sets in this definition implies that a fuzzy set $s(e) \in L^{U}$, where $e \in E$, represents a fuzzy evaluation of how individual objects in $U$ correspond to the parameter $e$ in $E$, that is, $s(e)(u) \in L$ is an $L$-valued measure of how the object $u \in U$ corresponds to parameter $e \in E$. We now present an example of an application of fuzzy soft sets in image processing.

Example 1. (Color image segmentation as a fuzzy soft set)

Let $(U, d)$ represent a finite metric space of pixels of a color image with metric $d$. For each pixel $u \in U$, we define a value $S(u)$ representing the color of $u$. This value can be represented in different ways. For instance, we can use the so-called HSV representation, that is, a vector $S(u)=[h, s, v]$, where $h$ represents the hue of the color, $s$ represents the saturation dimension, corresponding to various shades of brightly colored paint, and $v$ represents the value dimension, corresponding to a mixture with varying amounts of black or white paint. Accordingly, the color image can be represented as a mapping $S: U \rightarrow E=\left\{\mathbf{e}=[h, s, v]: h \in I_{h}, s \in I_{s}, v \in I_{v}\right\}$, where $I_{h}, I_{s}, I_{v}$ are the value sets of corresponding parameters.
However, we can use a different interpretation of $S$, particularly when we are interested in segmenting a given image because of its color. Let $E_{S}=S(U)=\{\mathbf{e} \in E: \exists u \in$ $U, S(u)=\mathbf{e}\} \subseteq E$. Then, we can consider a $[0,1]$-fuzzy soft set $\left(E_{S}, s\right)$ in the set $E$, where $s: E_{S} \rightarrow[0,1]^{U}$, and $s(\mathbf{e})(u)$ is a membership value that quantifies the grade of membership of the pixel $u \in U$ to the color segment represented by a color $\mathbf{e} \in E_{S}$. Evidently, a larger membership value implies that there are more pixels $v \in U$ with color $S(u)$, or a color similar to $S(u)$, in the immediate vicinity of pixel $u$. After a simplification, we can formally describe this situation as follows.

On the set $E$ of colors, we can define a fuzzy similarity relation $\sigma: E \times E \rightarrow[0,1]$ such that $\sigma\left(\mathbf{e}, \mathbf{e}^{\prime}\right)$ represents the similarity degree of two colors $\mathbf{e}, \mathbf{e}^{\prime} \in E$. Analogously, using the metric space $(U, d)$ of pixels, we can define another fuzzy similarity relation $\rho: U \times U \rightarrow[0,1]$ such that $\rho(u, v)$ expresses the fact that the pixels $u$ and $v$ are "similar," that is, they are close to each other. Then, the grade of membership $s(\mathbf{e})(u)$ of the pixel $u \in U$ to the color segment represented by the color e can be described by the value

$$
\mathbf{e} \in E_{S}, u \in U, s(\mathbf{e})(u)=\frac{\sum_{v \in U} \rho(u, v), \sigma(S(v), \mathbf{e})}{\sum_{v \in U} \rho(u, v)},
$$

which defines how true it is that in the vicinity of pixel $u$, there are sufficiently many pixels with a color similar to e.

Therefore, from this perspective, the color segmentation of an image $S$ can be identified with the fuzzy soft set $\left(E_{S}, s\right)$ in a set $E$, or with the $\alpha$-cut $\left(E_{S}, s\right)(\mathbf{e})_{\alpha}=\{u \in U: s(\mathbf{e})(u) \geq \alpha\}$ of a color segmentation corresponding to the color $\mathbf{e}$. More details regarding this application can be found in [22].

As mentioned in Introduction, powerset theories are widely used in algebra, logic, topology, and computer science. Numerous studies have been concerned with Zadeh's extension and its generalizations, which could be considered the first example of a powerset operator in fuzzy set theory. The theoretical justification of Zadeh's extension principle was first presented by Rodabaugh [18].

As general powerset theories and monads are not commonly used in the community of readers interested in fuzzy set structures, we repeat the basic definitions of these notions. For more details on category theory, readers are referred to, for example, [23]. The following definition by Rodabaugh [18] introduces a $C S L A T$ - powerset theory. If $f: X \rightarrow Y$ and $g: Y \rightarrow Z$ are morphisms in a category, $g . f: X \rightarrow Z$ denotes their composition. Morphisms in a category $\mathbf{K}$ are sometimes 
abbreviated as $\mathbf{K}$-morphisms.

Definition 2. Let $\mathbf{K}$ be a category. Then, $\mathbf{T}=(M, \rightarrow, V, \eta)$ is called a $C S L A T$-powerset theory in $\mathbf{K}$ if

1) $T: \mathbf{K} \rightarrow C S L A T$ is an object-mapping.

2) For each morphism $f: A \rightarrow B$ in $\mathbf{K}$, there exists a morphism $f_{\mathbf{T}}: T(A) \rightarrow T(B)$ in the category $C S L A T$.

3) $V: \mathbf{K} \rightarrow \mathbf{S e t}$ is a functor.

4) For each object $X \in \mathbf{K}, \eta$ determines in Set a mapping $\eta_{X}: V(X) \rightarrow W T(X)$, where $W: C S L A T \rightarrow$ Set is a forgetful functor.

5) For each $f: X \rightarrow Y$ in $\mathbf{K}$, we have $W\left(f_{\mathbf{T}}\right) \cdot \eta_{X}=$ $\eta_{Y} \cdot V(f)$

For simplicity, a $C S L A T$-powerset theory will be referred to as a powerset theory. A set $T(A)$ is called a powerset object of $X \in \mathbf{K}$. Let us consider the following standard examples.

Example 2. The powerset theory $\mathbf{P}=(P, \rightarrow, i d, \rho)$ in the category Set is defined as follows:

1) The object function $P$ : Set $\rightarrow C S L A T$ is defined by $P(X)=\left(2^{X}, \subseteq\right)$.

2) For each mapping $f: X \rightarrow Y$ in Set, $f_{P}: 2^{X} \rightarrow 2^{Y}$ is defined by $f_{P}(A)=f(A), A \in 2^{X}$.

3) For each $X \in$ Set, $\rho_{X}: X \rightarrow 2^{X}$ is defined by $\rho_{X}(x)=$ $\{x\} \in 2^{X}$.

Example 3. The powerset theory $\mathbf{Z}=(Z, \rightarrow, i d, \chi)$ in the category Set is defined as follows:

1) The object function $Z$ : Set $\rightarrow C S L A T$ is defined by $Z(X)=\left(L^{X}, \leq\right)$, where $\leq$ is the pointwise ordering derived from a lattice $L \in \mathcal{C}$.

2) For each mapping $f: X \rightarrow Y$ in Set, $f_{Z}: L^{X} \rightarrow L^{Y}$ is defined by

$$
\begin{aligned}
& s \in L^{X}, y \in Y, \\
& f_{Z}(s)(y)= \begin{cases}\bigvee_{x \in f^{-1}(y)} s(x), & f^{-1}(y) \neq \emptyset, \\
0_{L}, & \text { otherwise. }\end{cases}
\end{aligned}
$$

3) For each $X \in$ Set, $\chi_{X}: X \rightarrow L^{X}$ is the map such that $\chi_{X}(a)$ is the characteristic map of the singleton $\{a\}$ in the set $X$, that is,

$$
x \in X, \quad \chi_{X}(a)(x)= \begin{cases}1_{L}, & x=a, \\ 0_{L}, & \text { otherwise. }\end{cases}
$$

These basic powerset theories $\mathbf{P}, \mathbf{Z}$ are often used not only in mathematical constructions related to sets and fuzzy sets, but also in other fields, such as computer science and automata theory. In these cases, however, additional properties of these powerset theories are used, which are not derived from the general properties of powerset objects, but are based on special properties of the objects $L^{X}$. These special properties of some powerset objects (including $L^{X}$ ) can be summarized in monads and powerset theories defined by monads. Monads over a category have been used in fuzzifying mathematical objects, and there are several equivalent notions of a monad [19]. Their so-called clone form in a category $\mathbf{K}$ appears to be suitable for applications in both computer science and mathematics, and it intuitively expresses the basic notion of fuzzification. The use of monads for fuzzification is based on the extension of objects $X$ of a category $\mathbf{K}$ to another object $\widetilde{T}(X) \in \mathbf{K}$, which may be regarded as an object of a "cloud of fuzzy states" with a morphism $\eta: X \rightarrow \widetilde{T}(X)$ representing "crisp" states in the object of "fuzzy states." Then, a "fuzzy morphism" $f: X \rightsquigarrow Y$ is simply a morphism $f: X \rightarrow \widetilde{T}(Y)$ in the category $\mathbf{K}$, and a composition of fuzzy morphisms is defined by a special operation $\diamond$. The result of these constructions is a triple $\widetilde{\mathbf{T}}=(\widetilde{T}, \diamond, \eta)$, which Manes [19] called a fuzzy theory and is, in fact, a monad (or algebraic theory) in clone form. A system of objects of a category $\mathbf{K}$ with fuzzy morphisms and a composition $\diamond$ of fuzzy morphisms is then a Kleisli category in the category $\mathbf{K}$ ( [19]).

The following definition of a monad in clone form was introduced by Manes [19].

Definition 3. $\widetilde{\mathbf{T}}=(\widetilde{T}, \diamond, \eta)$ is a monad in a category $\mathbf{K}$ if

1) $\widetilde{T}: \mathbf{K} \rightarrow \mathbf{K}$ is an object function.

2) $\eta$ is a system of $\mathbf{K}$-morphisms $\eta_{A}: A \rightarrow \widetilde{T}(A)$ for any object $A$.

3) For each pair of K-morphisms $f: A \rightarrow \widetilde{T}(B)$ and $g$ $B \rightarrow \widetilde{T}(C)$, there exists a K-morphism (called a Kleisli composition) $g \diamond f: A \rightarrow \widetilde{T}(C)$ such that the operation $\diamond$ is associative.

4) For every K-morphism $f: A \rightarrow \widetilde{T}(B), \eta_{B} \diamond f=f$. 
5) $\diamond$ is compatible with composition of morphisms of $\mathbf{K}$, that is, for each pair of $\mathbf{K}$-morphisms $f: A \rightarrow B$ and $g: B \rightarrow$ $\widetilde{T}(C)$, we have $g \diamond\left(\eta_{B} \cdot f\right)=g . f$.

It should be noted that if $(\widetilde{T}, \diamond, \eta)$ is a monad in a category $\mathbf{K}$, then $\widetilde{T}: \mathbf{K} \rightarrow \mathbf{K}$ is a functor. In fact, for each morphism $f: A \rightarrow B$, the morphism $\widetilde{T}(f)$ is defined by

$$
\widetilde{T}(f)=\left(\eta_{B} \cdot f\right) \diamond 1_{\widetilde{T}(A)} .
$$

Moreover, $\eta$ represents identities on both sides for $\diamond$, that is, for each $A \rightarrow \widetilde{T}(B)$,

$$
\eta_{B} \diamond f=f, \quad f \diamond \eta_{A}=f
$$

In this case, $\eta: 1_{\mathbf{K}} \rightarrow \widetilde{T}$ is a natural transformation.

Let us recall some simple properties of Kleisli composition.

Lemma 1. Let $\widetilde{\mathbf{T}}=(\widetilde{T}, \diamond, \eta)$ be a monad in a category $\mathbf{K}$. Let $f: X \rightarrow Y, g: Y \rightarrow \widetilde{T}(Z), h: Z \rightarrow \widetilde{T}(U), e: \widetilde{T}(U) \rightarrow$ $W$ be morphisms in $\mathbf{K}$. Then,

$$
\begin{aligned}
& h \diamond(g . f)=(h \diamond g) . f, \quad e .(h \diamond g)=(e . h) \diamond g, \\
& \eta_{z} \diamond g=g \diamond \eta_{y} .
\end{aligned}
$$

We now present well-known examples of monads in the category Set.

Example 4. The structure $\widetilde{\mathbf{P}}=(\widetilde{P}, \diamond, \rho)$ is defined as follows:

1) For each object $X \in \operatorname{Set}, \widetilde{P}(X)=2^{X}$.

2) For each object $X \in$ Set, $\eta_{X}: X \rightarrow \widetilde{P}(X)$ is defined by $\rho_{X}(x)=\{x\}$.

3) For each $f: X \rightarrow \widetilde{P}(Y), g: Y \rightarrow \widetilde{P}(Z), g \diamond f: X \rightarrow$ $\widetilde{P}(Z)$ is defined by

$$
(g \diamond f)(x)=\bigcup_{y \in f(x)} g(y)
$$

Example 5. Let $L$ be a complete lattice with a t-norm $\otimes$. The structure $\widetilde{\mathbf{Z}}=(\widetilde{Z}, \diamond, \chi)$ is defined as follows:

1) For each object $X \in$ Set, the object function $\widetilde{Z}:$ Set $\rightarrow$ Set is defined by $\widetilde{Z}(X)=L^{X}$.

2) For each $X \in$ Set, $\chi_{X}: X \rightarrow L^{X}$ is the map such that $\chi_{X}(a)$ is the characteristic map of the singleton $\{a\}$ in the set $X$.
3) For each $f: X \rightarrow \widetilde{Z}(Y)$ and $g: Y \rightarrow \widetilde{Z}(Q)$ in Set, $g \diamond f: X \rightarrow \widetilde{Z}(Q)$ is defined by

$$
x \in X, z \in Q, \quad[(g \diamond f)(x)](z)=\bigvee_{y \in Y} f(x)(y) \otimes g(y)(z)
$$

As mentioned previously, some powerset theories have additional properties related to special properties of fuzzy sets. Examples of such theories are powerset theories in a category defined by monads. We present a general definition of such a powerset theory.

Definition 4. Let $\mathbf{T}=(T, \rightarrow, V, \chi)$ be a powerset theory in a category $\mathbf{K}$. Then, $\mathbf{T}$ is defined by a monad $\widetilde{\mathbf{T}}=(\widetilde{T}, \diamond, \eta)$ in $\mathbf{K}$ (or equivalently, the monad $\widetilde{\mathbf{T}}$ defines a powerset theory $\mathbf{T}$ ) if the following diagram of functors commutes:

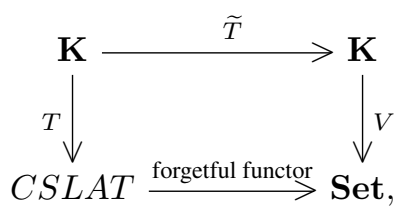

where for an arbitrary K-morphism $f: X \rightarrow Y, T(f)$ and $\widetilde{T}(f)$ are defined as follows:

$$
T(f)=f_{T}^{\rightarrow}, \quad \widetilde{T}(f)=\left(\eta_{Y} \cdot f\right) \diamond 1_{\widetilde{T}(X)} .
$$

Let us consider the following simple example of a powerset theory defined by a monad.

Example 6. The powerset theory $\mathbf{Z}$ is defined by the monad $\widetilde{\mathbf{Z}}$. In fact, by a simple calculation, for a map $f: X \rightarrow Y$, we obtain

$$
\begin{aligned}
s \in Z(X), & y \in Y, \\
Z(f)(s)(y) & =f_{Z}(s)(y)=\bigvee_{x \in X, f(x)=y} s(x) \\
& =\left(\chi_{Y} \cdot f\right) \diamond 1_{\widetilde{Z}(X)}(s)(y)=\widetilde{Z}(s)(y),
\end{aligned}
$$

and the diagram from Definition 4 commutes. Hence, $\mathbf{Z}$ is defined by $\widetilde{\mathbf{Z}}$.

It is also clear that the powerset theory $\mathbf{P}$ is defined by the $\operatorname{monad} \widetilde{\mathbf{P}}$.

\section{Powerset Theory of Fuzzy Soft Sets}

Herein, we show that, as classical fuzzy sets in a set $X$ define Zadeh's powerset theory with objects $Z(X)$ in the category Set, fuzzy soft sets define another powerset theory in another 
category. This powerset theory is a variant of the variable-basis fuzzy set powerset theory that Rodabaugh [18] introduced. The ground category for the variable-basis fuzzy set powerset theory is the category Set $\times \mathcal{C}$, where Set is the standard category of sets and mappings, and $\mathcal{C}$ is the category of appropriate complete lattices with the corresponding homomorphisms. We recall the construction of this powerset theory.

Let the object function $W:$ Set $\times \mathcal{C} \rightarrow C S L A T$ and the functor $V:$ Set $\times \mathcal{C} \rightarrow$ Set be defined by

$$
\begin{aligned}
& W(X, L)=\{f \mid f: X \rightarrow L\}=\left(L^{X}, \leq\right), \\
& V(X, L)=X, V(f, \varphi)=f
\end{aligned}
$$

where $\leq$ is the pointwise ordering, and for an arbitrary morphism $(f, \varphi):(X, L) \rightarrow(Y, M)$, the powerset extension $(f, \varphi)_{W}^{\rightarrow}: W(X, L) \rightarrow W(Y, M)$ is defined by

$$
\begin{aligned}
& s \in L^{X}, y \in Y, \\
& (f, \varphi)_{W}^{\rightarrow}(s)(y)= \begin{cases}\bigvee_{x \in X, f(x)=y} \varphi(s(x)), & f^{-1}(y) \neq \emptyset \\
0_{M}, & \text { otherwise }\end{cases}
\end{aligned}
$$

Moreover, for $(X, L)$, the map $\chi_{(X, L)}: X \rightarrow W(X, L)$ is defined by

$$
x, z \in X, \quad \chi_{(X, L)}(x)(z)= \begin{cases}1_{L}, & \text { iff } x=z, \\ 0_{L}, & \text { otherwise },\end{cases}
$$

The following theorem ensuring the existence of variable-base fuzzy set powerset theory was proven by Rodabaugh [24].

Theorem 1. Let $\mathcal{C}$ be the category of complete lattices with complete lattice-preserving homomorphisms. Then, $\mathbf{W}=$ $(W, \rightarrow, V, \chi)$ is a CSLAT-powerset theory in the category Set $\times \mathcal{C}$. This powerset theory is called Zadeh's variable-basis fuzzy set powerset theory.

As we showed in Example 6 the classical fixed-basis Zadeh's power set theory $\mathbf{Z}$ is defined by a monad. By a simple modification of the proof, it can be proven that Zadeh's variable-basis powerset theory $\mathbf{W}$ is also defined by a suitable monad in the category Set $\times \mathcal{C}$. For simplicity, we prove a variant in which the category $\mathcal{C}$ consists of complete residuated lattices, although it can also be proven under slightly weaker conditions.

Proposition 1. Let $\mathcal{C}$ be the category of complete residuated lattices. Then, there exists a monad $\widetilde{\mathbf{W}}=(\widetilde{W}, \triangle, \nu)$ in the category Set $\times \mathcal{C}$ such that $\widetilde{\mathbf{W}}$ defines the powerset theory $\mathbf{W}$.

Proof. Let the object function $\widetilde{W}:$ Set $\times \mathcal{C} \rightarrow$ Set $\times \mathcal{C}$ be defined by

$$
(X, L) \in \operatorname{Set} \times \mathcal{C}, \widetilde{W}(X, L)=(W(X, L), L) \in \operatorname{Set} \times \mathcal{C} .
$$

For $(X, L)$, we set $\nu_{(X, L)}=\left(\chi_{X}, i d_{L}\right):(X, L) \rightarrow \widetilde{W}(X, L)$, where $\chi_{X}$ is the characteristic function of subsets in $X$. Finally, for two morphisms $(f, \varphi):(X, L) \rightarrow \widetilde{W}(Y, M)$ and $(g, \psi)$ : $(Y, M) \rightarrow \widetilde{W}(Z, N)$, we set

$$
\begin{aligned}
& (g, \psi) \triangle(f, \varphi)=(g \diamond f, \psi \cdot \varphi) \\
& g \diamond f: X \rightarrow W(Z, N), \\
& x \in X, z \in Y, \quad g \diamond f(x)(z)=\bigvee_{y \in Y} \psi(f(x)(y)) \otimes g(y)(z)
\end{aligned}
$$

By a simple computation, it can be proven that the operation $\triangle$ is associative, and that the following equalities hold:

$$
\begin{aligned}
& \forall(f, \varphi):(X, L) \rightarrow(Y, M),(g, \psi):(Y, M) \rightarrow \widetilde{W}(Z, N), \\
& \nu_{(Y, M)} \triangle(f, \varphi)=(f, \varphi), \\
& (g, \psi) \cdot(f, \varphi)=(g, \psi) \triangle\left(\chi_{Y}, i d_{M}\right) \cdot(f, \varphi),
\end{aligned}
$$

by the identity $g \diamond\left(\chi_{Y} . f\right)=\left(g \diamond \chi_{Y}\right) . f=g . f$. Hence, $\widetilde{\mathbf{W}}$ is a monad in the category Set $\times \mathcal{C}$.

We now show that the powerset theory $\mathbf{W}$ is defined by $\widetilde{\mathbf{W}}$. To this end, we should prove that the following diagram commutes:

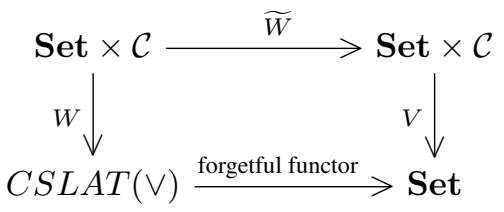

where for an arbitrary morphism $(f, \varphi):(X, L) \rightarrow(Y, M)$,

1) $W(f, \varphi):=(f, \varphi) \underset{W}{\vec{W}}$,

2) $\widetilde{W}(f, \varphi)=\left(\nu_{(Y, M)} \cdot(f, \varphi) \triangle 1_{\widetilde{W}(X, L)} \cdot\right.$

We have $V \widetilde{W}(f, \varphi)=V\left(\chi_{Y} \cdot f \diamond 1_{W(X, L)}, i d_{L}\right)=\chi_{Y} \cdot f \diamond$ $1_{W(X, L)}$, and for arbitrary $s \in W(X, L), y \in Y$, we obtain

$$
\begin{aligned}
& \chi_{Y} \cdot f \diamond 1_{W(X, L)}(s)(y) \\
& =\bigvee_{x \in X} \varphi\left(1_{W(X, L)}(s)(x)\right) \otimes \chi_{Y}(f(x))(y) \\
& =(f, \varphi)_{X}(s)(y) .
\end{aligned}
$$

Therefore, $\mathbf{W}$ is defined by the monad $\widetilde{\mathbf{W}}$.

to study the properties of powerset objects of fuzzy soft sets, Definition 11 implies that we cannot define them for objects 
of the category Set $\times \mathcal{C}$, but we must use a "richer" category. Instead of the category $\operatorname{Set} \times \mathcal{C}$, we consider some subcategories of $\operatorname{Set} \times \operatorname{Set} \times \mathcal{C}$. This reduction of the category $\operatorname{Set} \times \operatorname{Set} \times \mathcal{C}$ involves two steps. In the first, we construct a fuzzy soft set powerset theory analogous to the classical fuzzy set powerset theory. In this step, instead of the category Set $\times \operatorname{Set} \times \mathcal{C}$, we use the subcategory Set $\times \operatorname{Set}_{0} \times \mathcal{C}$, where $\operatorname{Set}_{0}$ is the subcategory of Set with surjective morphisms. In the second step, we define a monad, which in turn generates the fuzzy soft set powerset theory, as in the case of the classical fuzzy set powerset theory, which is defined by monad. We use the subcategory Set $\times \operatorname{Set}_{1} \times \mathcal{C}$, where $\operatorname{Set}_{1}$ is the subcategory with bijective maps as morphisms.

This restriction of the category Set to the subcategory Set $_{1}$ is based on several considerations concerning both the analogy of fuzzy soft sets with classical fuzzy sets, and the most common types of relationship (i.e., morphisms) among fuzzy soft sets. In the next part of the paper, we show that, as expected, any $L$-valued fuzzy set in the basic space $(X, L)$, that is, a map $s: X \rightarrow L$, can be identified with a fuzzy soft set in the space $(X,\{*\}, L)$, that is, with the map $\bar{s}: X \rightarrow L^{\{*\}}$. Hence, morphisms $(f, \varphi)$ in the basic space Set $\times \mathcal{C}$ for variable-basis fuzzy set powerset theory, which are used to derive relationships between fuzzy sets, can be identified with morphisms $\left(f, 1_{\{*\}}, \varphi\right)$ in $\mathbf{S e t} \times \mathbf{S e t}_{1} \times \mathcal{C}$.

Analogously, the relationships between fuzzy soft sets are derived from the morphisms between the corresponding objects of the basic spaces of the fuzzy soft sets, that is, from morphisms $(f, g, \varphi):(X, U, L) \rightarrow(Y, V, M)$. A typical example of such a relationship between fuzzy soft sets may be a change in the value structures by which we evaluate individual objects, that is, instead of a lattice $L$ we use another lattice $M$, but the evaluated objects remain unchanged. In this case, we use a morphism $\left(1_{X}, 1_{U}, \varphi\right)$ from Set $\times \mathbf{S e t}_{1} \times \mathcal{C}$. We can proceed analogously if we change the criteria from the set $X$ and replace them with other criteria. The morphism that we use in this case is $\left(f, 1_{U}, \varphi\right)$. In all these cases, we use morphisms from the category Set $\times \operatorname{Set}_{1} \times \mathcal{C}$.

Under these assumptions, we will show that, as in variablebasis fuzzy set powerset theory, fuzzy soft sets define a variablebase powerset theory, and the corresponding powerset theory is also defined by a monad.

In [11], the set of all $L$-valued fuzzy soft sets in a space $(X, U)$ was introduced, and an extension of maps between two spaces $(X, U)$ and $(Y, V)$ to maps between the corresponding sets of fuzzy soft sets was defined. Additional properties of extended maps between powerset objects of fuzzy soft sets were also investigated in [10]. To compare the relationships between fuzzy sets and fuzzy soft sets in the variable-basis case, we should define sets of variable-basis powerset objects of objects from the category Set $\times \operatorname{Set}_{0} \times \mathcal{C}$ sets and extensions of the corresponding morphisms. The sets of variable-basis powerset objects can be defined by the object function $T$ : Set $\times \operatorname{Set}_{0} \times \mathcal{C} \rightarrow C S L A T$, where

$$
\begin{aligned}
& (X, U, L) \in \mathbf{S e t} \times \mathbf{S e t}_{0} \times \mathcal{C}, \\
& T(X, U, L)=\left\{(E, s) \mid E \subseteq X, s: E \rightarrow L^{U}\right\} .
\end{aligned}
$$

According to Definition 1, the elements $(E, s)$ of the set $T(X, U$, $L)$ are called fuzzy soft sets in a space $(X, U, L)$. In the next theorem, we show that this object function is an object function of a $C S L A T$-powerset theory in the category $\operatorname{Set} \times \operatorname{Set}_{0} \times \mathcal{C}$.

Theorem 2. Let $\mathcal{C}$ be the category of complete lattices with complete lattice homomorphisms. The object function $T$ can be extended to the $C S L A T$-powerset theory $\mathbf{T}=(T, \rightarrow, R, \eta)$ in the category Set $\times \operatorname{Set}_{0} \times \mathcal{C}$, called the variable-basis fuzzy soft set powerset theory of fuzzy soft sets.

Proof. Let $(X, U, L)$ be an object in the category Set $\times$ $\operatorname{Set}_{0} \times \mathcal{C}$. In the set $T(X, U, L)$, we define an order relation by

$$
\begin{aligned}
& (E, s),(F, t) \in T(X, U, L), \\
& (E, s) \preceq(F, t) \text { iff } E \subseteq F,(\forall e \in E) s(e) \leq t(e) \text { in } L^{U} .
\end{aligned}
$$

It is clear that $T(X, U, L)$ is a complete $\bigvee$-semilattice with respect to this ordering, where for a system $\left\{\left(E_{i}, s_{i}\right): i \in\right.$ $I\} \subseteq T(X, U, L)$, we have

$$
\begin{aligned}
& \bigvee_{i \in I}\left(E_{i}, s_{i}\right)=\left(\bigcup_{i \in I} E_{i}, s\right), s: \bigcup_{i \in I} E_{i} \rightarrow L^{U}, \\
& x \in \bigcup_{i \in I} E_{i}, s(x)=\bigvee_{j \in I, x \in E_{j}} s_{j}(x) .
\end{aligned}
$$

Let $R: \mathbf{K}=$ Set $\times$ Set $_{0} \times \mathcal{C} \rightarrow$ Set be a forgetful functor defined by $R(X, U, L)=X, R(f, g, \varphi)=f$.

For an arbitrary object $(X, U, L)$ and $x, z \in X$, we set

$$
\begin{aligned}
& \eta_{(X, U, L)}: X \rightarrow T(X, U, L), \quad \eta_{(X, U, L)}(x)=\left(\{x\}, \eta_{x}\right), \\
& \eta_{x}:\{x\} \rightarrow L^{U}, \eta_{x}(x)=\underline{1}_{L},
\end{aligned}
$$

where $\underline{1}_{L}, \underline{0}_{L}$ are constant fuzzy sets in $U$ with values $1_{L}, 0_{L}$, respectively, and for a K-morphism $(f, g, \varphi):(X, U, L) \rightarrow$ 
$(Y, V, M)$, we set

$$
\begin{aligned}
& (f, g, \varphi)_{T}^{\rightarrow}: T(X, U, L) \rightarrow T(Y, V, M), \\
& (E, s) \in T(X, U, L), \quad(f, g, \varphi)_{T}(E, s)=\left(f(E), s^{\rightarrow}\right), \\
& s \rightarrow: f(E) \rightarrow M^{V}, \\
& y \in f(E), v \in V, \\
& s \rightarrow(y)(v)=\bigvee_{u \in g^{-1}(v)\left\{z \in f^{-1}(y) \cap E\right\}} \varphi(s(z)(u)),
\end{aligned}
$$

for all $(E, s) \in T(X, U, L), y \in f(E), v \in V$. As $y \in f(E)$, we have $f^{-1}(y) \cap E \neq \emptyset$, and therefore $(f, g, \varphi)_{T}$ is well defined. It should be mentioned that the map $(f, g, \varphi) \vec{T}$ represents a variable-basis extension of a map introduced in [11]. As $\varphi$ from a morphism $(f, g, \varphi)$ is a complete lattice homomorphism, by a simple computation, we can prove that $(f, g, \varphi)_{T}$ is a morphism in the category $C S L A T$. For a morphism $(f, g, \varphi):(X, U, L) \rightarrow(Y, V, M)$, let us consider the following diagram:

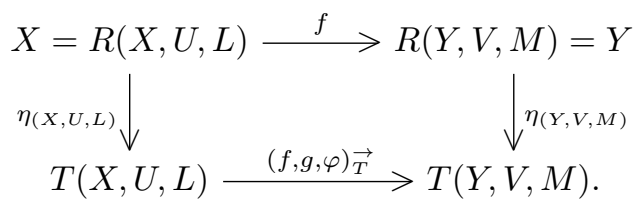

We prove that this diagram commutes. In fact, as $g$ is a surjective map, for $x \in X, v \in V$, we have

$$
\begin{gathered}
(f, g, \varphi)_{T}^{\vec{T}}\left(\eta_{(X, U, L)}(x)\right)=(f, g, \varphi)_{T}\left(\{x\}, \eta_{x}\right) \\
=\left(\{f(x)\}, \eta_{x}\right), \\
\eta_{x}^{\rightarrow}(f(x))(v)=\bigvee_{u \in g^{-1}(v) z \in f^{-1}(f(x)) \cap\{x\}} \varphi\left(\eta_{x}(z)(u)\right) \\
=1_{M}=\eta_{\{f(x)\}}(f(x)) .
\end{gathered}
$$

Hence, $\left(\{f(x)\}, \eta_{x}\right)=\left(\{f(x)\}, \eta_{\{f(x)\}}\right)$, and the diagram commutes. Therefore, $\mathbf{T}$ is a CSLAT-powerset theory.

We prove that, as in a variable-basis fuzzy set powerset theory, the variable-basis fuzzy soft set powerset theory is defined by a monad. However, to prove this, we should consider the restriction of the powerset theory $\mathbf{T}$ to the subcategory $\mathbf{S e t} \times \operatorname{Set}_{1} \times \mathcal{C}$ and a monad in this subcategory. Under this assumption, we prove that this restriction (which, for simplicity, is denoted again by $\mathbf{T}$ ) is actually defined by a monad. As seen in the next section, this restriction does not disrupt the generality of the relationship between the fuzzy soft set powerset theory and fuzzy set powerset theory. This is because (as follows from Proposition 2, the transformation of classical fuzzy sets into fuzzy soft sets takes place in the base space $\mathbf{S e t} \times \mathbf{S e t}_{1} \times \mathcal{C}$ and uses this reduced fuzzy soft sets powerset theory.

Unlike the proof of this statement in the case of fuzzy sets, the proof for fuzzy soft sets is more complicated.

Theorem 3. Let $\mathcal{C}$ be the category of complete residuated lattices. There exists a monad $\widetilde{\mathbf{T}}=(\widetilde{T}, \odot, \xi)$ in the category Set $\times$ Set $_{1} \times \mathcal{C}$ such that the variable-basis fuzzy soft set powerset theory $\mathbf{T}$ is defined by $\widetilde{\mathbf{T}}$.

Proof. Let the object function $\widetilde{T}:$ Set $\times$ Set $_{1} \times \mathcal{C} \rightarrow$ Set $\times \operatorname{Set}_{1} \times \mathcal{C}$ be defined by

$$
\begin{aligned}
& (X, U, L) \in \text { Set } \times \text { Set }_{0} \times \mathcal{C}, \\
& \widetilde{T}(X, U, L)=(T(X, U, L), U, L),
\end{aligned}
$$

where $T(X, U, L)$ is identified with the underlying set of a complete lattice from Theorem 2 Let the morphism $\xi_{(X, U, L)}$ be defined by

$$
\begin{aligned}
& \xi_{(X, U, L)}:(X, U, L) \rightarrow \widetilde{T}(X, U, L), \\
& \xi_{(X, U, L)}=\left(\eta_{(X, U, L)}, 1_{U}, 1_{L}\right),
\end{aligned}
$$

where $\eta_{(X, U, L)}$ is from Theorem 2 For morphisms

$$
\begin{aligned}
& (f, k, \varphi):(X, U, L) \rightarrow \widetilde{T}(Y, V, M), \\
& (g, j, \psi):(Y, V, M) \rightarrow \widetilde{T}(Z, W, N),
\end{aligned}
$$

we define the composition

$$
\begin{aligned}
& (g, j, \psi) \odot(f, k, \varphi) \\
& =(g \diamond f, j . k, \psi \cdot \varphi):(X, U, L) \rightarrow \widetilde{T}(Z, W, N),
\end{aligned}
$$

where $\diamond$ is a composition map that is defined as follows. If $f: X \rightarrow T(Y, V, M)$ is a map, then for $x \in X$, we use the notation

$$
f(x)=\left(Y_{x}^{f}, f_{x}\right) \in T(Y, V, M)
$$

where $\emptyset \neq Y_{x}^{f} \subseteq Y$ and $f_{x}: Y_{x}^{f} \rightarrow M^{V}$. Then, for maps $f: X \rightarrow T(Y, V, M)$ and $g: Y \rightarrow T(Z, W, N)$, we define the composition map $g \diamond f: X \rightarrow T(Z, W, N)$ by

$$
\begin{aligned}
& x \in X, g \diamond f(x)=\left(Z_{x}^{g \diamond f},(g \diamond f)_{x}\right) \in T(Z, W, N), \\
& Z_{x}^{g \diamond f}=\bigcup_{y \in Y_{x}^{f}} Z_{y}^{g}, \quad(g \diamond f)_{x}: Z_{x}^{g \diamond f} \rightarrow N^{W}, \\
& a \in Z_{x}^{g \diamond f}, w \in W,
\end{aligned}
$$




$$
(g \diamond f)_{x}(a)(w)=\bigvee_{\left\{y \in Y_{x}^{f}: a \in Z_{y}^{g}\right\}} \psi\left(f_{x}(y)\left(j^{-1}(w)\right)\right) \otimes g_{y}(a)(w)
$$

where

$$
\forall x \in X, \forall y \in Y_{x}^{f} \quad f(x)=\left(Y_{x}^{f}, f_{x}\right), \quad g(y)=\left(Z_{y}^{g}, g_{y}\right)
$$

$\diamond$ is well defined because $j$ is a bijection. We now prove that $\widetilde{\mathbf{T}}=(\widetilde{T}, \odot, \xi)$ is a monad in the category $\mathbf{S e t} \times \mathbf{S e t}_{1} \times \mathcal{C}$. In fact, let

$$
\begin{aligned}
& (f, k, \varphi):(X, U, L) \rightarrow \widetilde{T}(Y, V, M), \\
& (g, j, \psi):(Y, V, M) \rightarrow \widetilde{T}(Z, W, N), \\
& (h, m, \omega):(Z, W, N) \rightarrow \widetilde{T}(R, Q, S) .
\end{aligned}
$$

We prove that the operation $\odot$ is associative. We have

$$
\begin{aligned}
& (h, m, \omega) \odot((g, j, \psi) \odot(f, k, \varphi)) \\
& =(h \diamond(g \diamond f), m \cdot j \cdot k, \omega \cdot \psi \cdot \varphi), \\
& ((h, m, \omega) \odot(g, j, \psi)) \odot(f, k, \varphi) \\
& =((h \diamond g) \diamond f, m \cdot j . k, \omega \cdot \psi \cdot \varphi),
\end{aligned}
$$

and we need only prove that $h \diamond(g \diamond f)=(h \diamond g) \diamond f: X \rightarrow$ $T(R, Q, S)$. Let $x \in X$. Then, using relations (2), (3), and (4), we obtain

$$
\begin{aligned}
(h \diamond(g \diamond f))(x)= & \left(R_{x}^{h \diamond(g \diamond f)},(h \diamond(g \diamond f))_{x}\right) \\
= & \left(\bigcup_{z \in Z_{x}^{g \diamond f}} R_{z}^{h},(h \diamond(g \diamond f))_{x}\right) \\
= & \left(\bigcup_{z \in \bigcup_{y \in Y_{x}^{f}} Z_{y}^{g}} R_{z}^{h},(h \diamond(g \diamond f))_{x}\right), \\
((h \diamond g) \diamond f)(x)= & \left(R_{x}^{(h \diamond g) \diamond f},((h \diamond g) \diamond f)_{x}\right) \\
= & \left(\bigcup_{y \in Y_{x}^{f}} R_{y}^{h \diamond g},((h \diamond g) \diamond f)_{x}\right) \\
= & \left(\bigcup_{y \in Y_{x}^{f}} \bigcup_{z \in Z_{y}^{g}} R_{z}^{h},((h \diamond g) \diamond f)_{x}\right) .
\end{aligned}
$$

By a simple calculation, it can be proven that

$$
R_{x}^{h \diamond(g \diamond f)}=\bigcup_{z \in \bigcup_{y \in Y_{x}^{f}} Z_{y}^{g}} R_{z}^{h}=\bigcup_{y \in Y_{x}^{f}} \bigcup_{z \in Z_{y}^{g}} R_{z}^{h}=R_{x}^{(h \diamond g) \diamond f} .
$$

We prove that $((h \diamond g) \diamond f)_{x}=(h \diamond(g \diamond f))_{x}$. Using the notation (2), we obtain the following maps:

$$
\begin{aligned}
& x \in X, \quad f_{x}: Y_{x}^{f} \rightarrow M^{V}, \quad Y_{x}^{f} \subseteq Y, \\
& y \in Y, \quad g_{y}: Z_{y}^{g} \rightarrow N^{W}, \quad Z_{y}^{g} \subseteq Z, \\
& z \in Z, \quad h_{z}: R_{z}^{h} \rightarrow S^{Q}, \quad R_{z}^{h} \subseteq R, \\
& x \in X, \quad(g \diamond f)_{x}: Z_{x}^{g \diamond f} \rightarrow N^{W}, \quad Z_{x}^{g \diamond f} \subseteq Z, \\
& y \in Y, \quad(h \diamond g)_{y}: R_{y}^{h \diamond g} \rightarrow S^{Q}, \quad R_{y}^{h \diamond g} \subseteq R .
\end{aligned}
$$

For $a \in R_{x}^{h \diamond(g \diamond f)}, q \in Q$, using definition (5), we obtain

$$
\begin{aligned}
(h \diamond(g \diamond f))_{x}(a)(q) & \bigvee_{\left\{z \in Z_{x}^{g \diamond f}: a \in R_{z}^{h}\right\}} \omega\left((g \diamond f)_{x}(z)\left(m^{-1}(q)\right)\right) \otimes h_{z}(a)(q) \\
= & \bigvee_{\left\{z \in Z_{x}^{g \diamond f}: a \in R_{z}^{h}\right\}} \bigvee_{\left\{y \in Y_{x}^{f}: z \in Z_{y}^{g}\right\}}\left[\omega \cdot \psi\left(f_{x}(y)\left(j^{-1}(w)\right)\right)\right. \\
& \left.\otimes \omega\left(g_{y}(z)\left(m^{-1}(q)\right)\right) \otimes h_{z}(a)(q)\right] \\
= & \bigvee_{\left\{z \in \cup_{y \in Y_{x}^{f}} Z_{y}^{g}: a \in R_{z}^{h}\right\}} \bigvee_{\left\{y \in Y_{x}^{f}: z \in Z_{y}^{g}\right\}}\left[\omega \cdot \psi\left(f_{x}(y)\left(j^{-1}(w)\right)\right)\right. \\
& \left.\otimes \omega\left(g_{y}(b)\left(m^{-1}(q)\right)\right) \otimes h_{z}(a)(q)\right] \\
= & \bigvee_{(v, w, z, y) \in A} \Delta(v, w, z, y),
\end{aligned}
$$

where

$$
\begin{aligned}
& A=\{(v, w, z, y): j(v)=w, m(w)=q, \\
& \left.y \in Y_{x}^{f}, z \in Z_{y}^{g}, a \in R_{z}^{h}\right\}, \\
& \Delta(v, w, z, y)=\omega \cdot \psi\left(f_{x}(y)(v)\right) \otimes \omega\left(g_{y}(z)(w)\right) \otimes h_{z}(a)(q) .
\end{aligned}
$$

Analogously, we obtain

$$
\begin{aligned}
& ((h \diamond g) \diamond f)_{x}(a)(q) \\
& =\bigvee_{\left\{y \in Y_{x}^{f}: a \in R_{y}^{h \diamond g}\right\}} \omega \cdot \psi\left(f_{x}(y)\left((m \cdot j)^{-1}(q)\right)\right) \otimes(h \diamond g)_{y}(a)(q) \\
& =\bigvee_{\left\{y \in Y_{x}^{f}: a \in \bigcup_{z \in Z_{y}^{g}} R_{z}^{h}\right\}\left\{z \in Z_{y}^{g}: a \in R_{z}^{h}\right\}}\left[\omega \psi\left(f_{x}(y)\left((m \cdot j)^{-1}(q)\right)\right)\right. \\
& \left.\quad \otimes \omega\left(g_{y}(z)\left(m^{-1}(q)\right)\right) \otimes h_{z}(a)(q)\right] \\
& =\bigvee_{(v, w, z, y) \in B} \Delta(v, w, z, y),
\end{aligned}
$$

where

$$
\begin{aligned}
B= & \left\{(v, w, z, y): m(w)=q, m \cdot j(v)=q, y \in Y_{x}^{f},\right. \\
& \left.z \in Z_{y}^{g}, a \in R_{z}^{h}\right\} .
\end{aligned}
$$

As $j$ and $m$ are bijective maps, we obtain $A=B,(h \diamond(g \diamond f))_{x}$ $=((h \diamond g) \diamond f)_{x}$, and the operation $\diamond$ is associative. Thus, the 
operation $\odot$ is associative.

We show that for arbitrary morphism $(f, k, \varphi):(X, U, L) \rightarrow$ $\widetilde{T}(Y, V, M)$, the identity

$$
\begin{aligned}
\xi_{(Y, V, M)} \odot(f, k, \varphi) & =\left(\eta_{(X, U, L)}, 1_{V}, 1_{M}\right) \odot(f, k, j) \\
& =\left(\eta_{(X, U, L)} \oslash f, k, \varphi\right)=(f, k, \varphi)
\end{aligned}
$$

holds. In fact, from the definition of $\eta$ and relations (3) and (4), we obtain

$$
\begin{aligned}
& x \in X, \\
& \left(\eta_{(X, U, L)} \diamond f\right)(x)=\left(\bigcup_{y \in Y_{x}}\{y\}=Y_{x}^{f},\left(\eta_{(X, U, L)} \diamond f\right)_{x}\right), \\
& \left(\eta_{(X, U, L)} \diamond f\right)_{x}: Y_{x}^{f} \rightarrow M^{V}, \\
& a \in Y_{x}^{f}, v \in V, \\
& \left(\eta_{(X, U, L)} \diamond f\right)_{x}(a)(v)=f_{x}(a)(v) \otimes \eta_{a}(a)(v)=f_{x}(a)(v),
\end{aligned}
$$

and $\left(\eta_{(X, U, L)} \diamond f\right)(x)=\left(Y_{x}^{f},\left(\eta_{(X, U, L)} \diamond f\right)_{x}\right)=\left(Y_{x}^{f}, f_{x}\right)=$ $f(x)$.

We show that for morphisms $(f, k, \varphi):(X, U, L) \rightarrow(Y, V, M)$ and $(g, j, \psi):(Y, V, M) \rightarrow \widetilde{T}(Z, W, M)$, the following identity holds:

$$
(g, j, \psi) \odot\left(\xi_{(Y, V, M)},(f, k, \varphi)\right)=(g, j, \psi),(f, k, \varphi),
$$

In fact, we have

$$
\begin{aligned}
& (g, j, \psi) \odot\left(\xi_{(Y, V, M)},(f, k, \varphi)\right) \\
& =\left(g \diamond\left(\eta_{(Y, V, M)}, f\right), j . k, \psi \cdot \varphi\right) .
\end{aligned}
$$

Hence, we should prove that $g \diamond\left(\eta_{(Y, V, M)} . f\right)=g . f$. For simplicity, instead of $\eta_{(Y, V, M)}$, we write only $\eta$. For $x \in X$, according to relations (3) and (4), we have

$$
\begin{aligned}
& \eta \cdot f(x)=\eta(f(x))=\left(Y_{x}^{\eta \cdot f}=\{f(x)\}, \eta_{f(x)}\right), \\
& (g \diamond \eta \cdot f)(x)=\left(Z_{x}^{g \diamond \eta \cdot f},(g \diamond \eta \cdot f)_{x}\right), \\
& Z_{x}^{g \diamond \eta \cdot f}=\bigcup_{y \in Y_{x}^{\eta \cdot f}} Z_{y}^{g}=Z_{f(x)}^{g}, \\
& (g \diamond \eta \cdot f)_{x}: Z_{f(x)}^{g} \rightarrow N^{W} .
\end{aligned}
$$

Let $a \in Z_{f(x)}^{g}, w \in W$. Then, according to relation (5), we have

$$
\begin{aligned}
& (g \triangleright \eta \cdot f)_{x}(a)(w) \\
& =\bigvee_{\left\{y \in Y_{x}^{\eta \cdot f}\right.}\left(\eta \in Z_{y}^{g}\right\} \\
& (\eta \cdot f)_{x}(y)\left(j^{-1}(w)\right) \otimes g_{y}(a)(w)
\end{aligned}
$$

$$
\begin{aligned}
& =\eta_{f(x)}(f(x))\left(j^{-1}(w)\right) \otimes g_{f(x)}(a)(w)=g_{f(x)}(a) \\
& =(g \cdot f)_{x}(a)(w) .
\end{aligned}
$$

Furthermore, we have

$$
\begin{aligned}
(g . f)(x) & =g(f(x))=\left(Z_{f(x)}^{g}, g_{f(x)}\right) \\
& =\left(Z_{x}^{g \diamond \eta \cdot f},(g \diamond \eta \cdot f)_{x}\right) \\
& =(g \diamond \eta \cdot f)(x) .
\end{aligned}
$$

Hence, identity (6) holds, and we proved that $\widetilde{\mathbf{T}}=(\widetilde{T}, \odot, \xi)$ is a monad in the category Set $\times \operatorname{Set}_{1} \times \mathcal{C}$.

Finally, we prove that the monad $\widetilde{\mathbf{T}}$ defines the powerset theory $\mathbf{T}$. We should prove that the following functor diagram commutes:

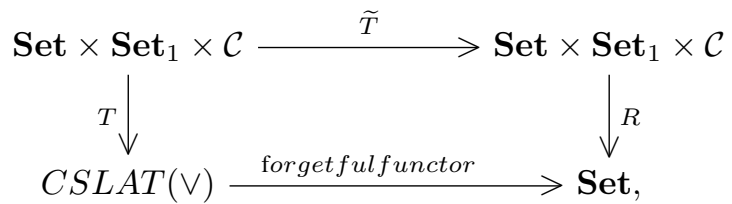

where for a morphism $(f, k, \varphi):(X, U, L) \rightarrow(Y, V, M)$ in the category $\operatorname{Set} \times \operatorname{Set}_{1} \times \mathcal{C}$,

$$
\begin{aligned}
T(f, k, \varphi)= & (f, k, \varphi)_{T}: T(X, U, L) \rightarrow T(Y, V, M), \\
\widetilde{T}(f, k, \varphi)= & \left(\xi_{(Y, V, M)} \cdot(f, k, \varphi)\right) \odot 1_{\widetilde{T}(X, U, L)}: \\
& \widetilde{T}(X, U, L) \rightarrow \widetilde{T}(Y, V, M) .
\end{aligned}
$$

It is clear that the diagram commutes for objects of the category. According to the definition of the powerset theory in Theorem 2, for $(E, s) \in T(X, U, L)$, we have

$$
\begin{aligned}
& (f, k, \varphi)_{T}(E, s)=\left(f(E), s^{\rightarrow}\right), \\
& R \widetilde{T}(f, k, \varphi)(E, s) \\
& =R\left(\left(\xi_{(Y, V, M)} \cdot(f, k, \varphi)\right) \odot 1_{\widetilde{T}(X, U, L)}\right)(E, s) \\
& =R\left(\left(\eta_{(Y, V, M)} \cdot f \diamond 1_{T(X, U, L)}, k, \varphi\right)\right)(E, s) \\
& =\left(\eta_{(Y, V, M)} \cdot f \diamond 1_{T(X, U, L)}\right)(E, s) .
\end{aligned}
$$

According to relations (3) and (4), we have

$$
\begin{aligned}
& \left(\eta . f \diamond 1_{T(X, U, L)}\right)(E, s) \\
& =\left(Y_{(E, s)}^{\left.\eta \cdot f \diamond 1_{T(X, U, L)}\right)},\left(\eta \cdot f \diamond 1_{T(X, U, L)}\right)_{(E, s)}\right), \\
& Y_{(E, s)}^{\eta \cdot f \diamond 1_{T(X, U, L)}}=\bigcup_{x \in E} Y_{x}^{\eta \cdot f}=\bigcup_{x \in E}\{f(x)\}=f(E), \\
& (E, s)=1_{T(X, U, L)}(E, s)=\left(X_{(E, s)}^{1_{T(X, U, L)}}, 1_{T(X, U, L),(E, s)}\right) .
\end{aligned}
$$


Therefore, for $a \in f(E), v \in V$, we obtain

$$
\begin{aligned}
& \left(\eta \cdot f \diamond 1_{T(X, U, L)}\right)_{(E, s)}(a)(v) \\
& =\bigvee_{\{z \in E \mid a=f(z)\}} \varphi\left(s(z)\left(k^{-1}(v)\right)\right) \otimes(\eta . f)_{z}(a)(v) \\
& =\bigcup_{\{z \in E \mid a=f(z)\}} \varphi\left(s(z)\left(k^{-1}(v)\right)\right) \otimes \eta_{a}(a)(v)=s^{\rightarrow}(a)(v) .
\end{aligned}
$$

Therefore, $R \widetilde{T}(f, k, \varphi)(E, s)=(f(E), s \rightarrow)=T(f, k, \varphi)(E, s)$, and the functor diagram commutes. Hence, $\mathbf{T}$ is defined by the $\operatorname{monad} \widetilde{\mathbf{T}}$.

As expected, any $L$-valued fuzzy set (i.e., an element of $W(X, L))$ can also be considered a fuzzy soft set in the space $(X,\{*\}, L)$, that is, an element of $T(X,\{*\}, L)$. In fact, if $s \in$ $W(X, L)$, then $s$ can be identified with $\left(E_{s},[s]\right) \in T(X,\{*\}, L)$, where $E_{s}=\left\{x \in X: s(x) \neq 0_{L}\right\}$ and $[s]: E_{s} \rightarrow L^{\{*\}}$ is defined by $[s](x)(*)=s(x) \in L$. Moreover, the embedding $W(X, L) \hookrightarrow T(X,\{*\}, L)$ can be extended to a more precisely defined relationship between $W$ and $T$, using the notion of a morphism between the powerset theories. The existence of a morphism between these powerset theories allows for a more precise understanding of the relationship between classical fuzzy sets and fuzzy soft sets.

The notion of a morphism of powerset theories is introduced in the following definition. We recall that the commutativity of the diagram

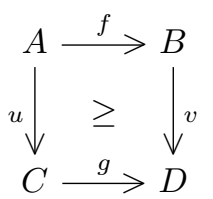

implies that $g . u \geq v . f$, where $D$ is an object with ordering $\leq$.

Definition 5. Let $\mathbf{T}=(T, \rightarrow, V, \eta)$ and $\mathbf{R}=(R, \Rightarrow, U, \tau)$ be $C S L A T$-powerset theories in the categories $\mathbf{K}$ and $\mathbf{J}$, respectively. Then, $(G, \Phi, \Psi):(\mathbf{K}, \mathbf{T}) \rightarrow(\mathbf{J}, \mathbf{R})$ is a morphism of $C S L A T$-powerset pairs if for each object $X \in \mathbf{K}$, we have the following:

1) $G: \mathbf{K} \rightarrow \mathbf{J}$ is a functor.

2) $\Phi_{X}: T(X) \rightarrow R(G(X))$ is a morphism in the category CSLAT.

3) $\Psi_{X}: V(X) \rightarrow U(G(X))$ is a map in the category Set.

4) For each morphism $f: X \rightarrow Y$ in $\mathbf{K}$, the following dia- grams commute:

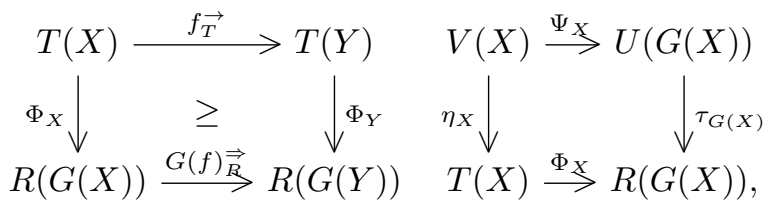

that is, $G(f) \Rightarrow . \Phi_{X} \geq \Phi_{Y} \cdot f_{T}^{\rightarrow}$, where $\leq$ is the ordering in the $C S L A T$ object $R(G(Y))$.

A morphism $(G, \Phi, \Psi)$ is called an embedding if $R: \mathbf{K} \hookrightarrow$ $\mathbf{J}$ is an embedding functor, $\Phi_{X}$ is a monomorphism in the category $C S L A T$, and $\Psi$ is an injective map.

We now prove that there is an embedding morphism between the powerset theories $\mathbf{W}$ and $\mathbf{T}$ that interprets the relationship between classical fuzzy sets and fuzzy soft sets.

Proposition 2. Let $\mathcal{C}$ be the category of complete lattices. Then, there exists an embedding morphism of CSLAT-powerset pairs

$$
(G, \Phi, \Psi):(\operatorname{Set} \times \mathcal{C}, \mathbf{W}) \rightarrow\left(\operatorname{Set} \times \operatorname{Set}_{1} \times \mathcal{C}, \mathbf{T}\right) .
$$

Therefore, the variable-basis fuzzy set powerset theory is a special case of the variable-basis fuzzy soft set powerset theory.

Proof. We define the functor $G: \operatorname{Set} \times \mathcal{C} \rightarrow \operatorname{Set} \times \operatorname{Set}_{1} \times \mathcal{C}$ by

$$
G(X, L)=(X,\{*\}, L), \quad G(f, \varphi)=\left(f, 1_{*}, \varphi\right),
$$

where $1_{*}:\{*\} \rightarrow\{*\}$. It is clear that $G$ is an embedding functor. For $(X, L) \in \operatorname{Set} \times \mathcal{C}$, we set

$$
\begin{aligned}
& \Phi_{(X, L)}: W(X, L) \hookrightarrow T(G(X, L))=T(X,\{*\}, L), \\
& s \in W(X, L), \quad \Phi_{(X, L)}(s)=\left(E_{s},[s]\right), \\
& E_{s}=\left\{z \in X: s(x) \neq 0_{L}\right\}, \quad[s]: E_{s} \rightarrow L^{\{*\}}, \\
& z \in E_{s}, \quad[s](z)(*)=s(z), \\
& \Psi_{(X, L)}=1_{X}: X \rightarrow X .
\end{aligned}
$$

It is clear that $\Phi_{(X, L)}$ is a complete $\bigvee$-semilattice homomorphism. In fact, we have

$$
\begin{aligned}
\Phi_{(X, L)}\left(\bigvee_{i \in I} s_{i}\right) & =\left(E_{\bigvee_{i \in I} s_{i}},\left[\bigvee_{i \in I} s_{i}\right]\right)=\left(\bigcup_{i \in I} E_{s_{i}}, \bigvee_{i \in I}\left[s_{i}\right]\right) \\
& =\bigvee_{i \in I} \Phi_{(X, L)}\left(s_{i}\right),
\end{aligned}
$$

where $\left[s_{i}\right]$ is considered an $L^{\{*\}}$-valued fuzzy set in $E_{\bigvee_{i} s_{i}}$. We show that the following diagram commutes: 


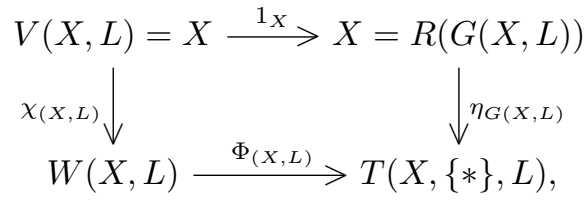

Let $x \in X$. We obtain

$$
\begin{aligned}
& \eta_{(G(X, L)}(x)=\left(\{x\}, \eta_{x}\right), \\
& \begin{aligned}
\eta_{x}:\{x\} \rightarrow L^{\{*\}}, \quad \eta_{x}(x)(*)=1_{L}, \\
\Phi_{(X, L)}\left(\chi_{(X, L)}(x)\right)=\left(E_{\chi_{(X, L)}(x)},\left[\chi_{(X, L)}(x)\right]\right) \\
=\left(\{x\},\left[\chi_{(X, L)}(x)\right]\right),
\end{aligned} \\
& {\left[\chi_{(X, L)}(x)\right](x)(*)=1_{L} .}
\end{aligned}
$$

Hence, the diagram commutes. We show that for an arbitrary morphism $(f, \varphi):(X, L) \rightarrow(Y, M)$, inequality holds in the diagram

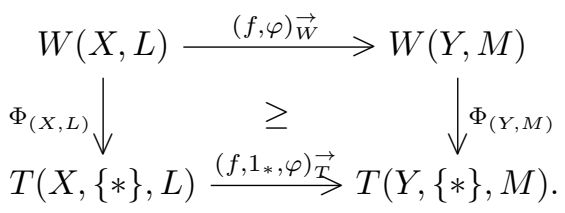

Let $s \in W(X, L)$. From the proof of Theorem 2, we have

$$
\begin{aligned}
& \left(f, 1_{*}, \varphi\right)_{T}\left(\phi_{(X, L)}(s)\right)=\left(f, 1_{*}, \varphi\right)_{T}\left(E_{s},[s]\right) \\
& =\left(f\left(E_{s}\right),[s]^{\rightarrow}\right), \\
& {[s] \rightarrow: f\left(E_{s}\right) \rightarrow M^{\{*\}},} \\
& f(x) \in f\left(E_{s}\right), \quad[s] \rightarrow(f(x))(*)=\bigvee_{z \in f^{-1}(f(x)) \cap E_{s}} \varphi(s(z)) .
\end{aligned}
$$

Moreover, we have

$$
\begin{aligned}
& \Phi_{(Y, M)}((f, \varphi) \underset{W}{\vec{W}}(s))=\left(F, t^{\rightarrow}\right), \\
& F=\left\{y \in Y:(f, \varphi)_{W}(s)(y) \neq 0_{M}\right\} \\
& =\left\{y \in Y: f^{-1}(y) \neq \emptyset, \bigvee_{x \in f^{-1}(y)} \varphi(s(x)) \neq 0_{M}\right\}, \\
& t^{\rightarrow}: F \rightarrow M^{\{*\}}, \\
& y \in F, t^{\rightarrow}(y)(*)=(f, \varphi)_{W}^{\rightarrow}(s)(y)=\bigvee_{x \in f^{-1}(y)} \varphi(s(x)) \\
& =\bigvee_{x \in f^{-1}(y) \cap E_{s}} \varphi(s(x)) .
\end{aligned}
$$

As $F \subseteq f\left(E_{s}\right)$, for $f(x) \in F$, we obtain

$$
t^{\rightarrow}(f(x))(*)=\bigvee_{z \in f^{-1}(f(x)) \cap E_{s}} \varphi(s(z))=[s] \rightarrow(f(x))(*),
$$

and $(F, t \rightarrow) \preceq\left(f\left(E_{s}\right),[s] \rightarrow\right)$. Therefore,

$$
(G, \Phi, \Psi):(\operatorname{Set} \times \mathcal{C}, \mathbf{W}) \rightarrow\left(\operatorname{Set} \times \operatorname{Set}_{1} \times \mathcal{C}, \mathbf{T}\right)
$$

is a morphism of powerset pairs.

In Proposition 2 we showed that any fuzzy set can be considered a special fuzzy soft set. In the next proposition, we prove the converse: Any fuzzy soft set can be extended to a fuzzy set, and this extension process is a morphism from the powerset theory $\mathbf{T}$ to the powerset theory $\mathbf{W}$.

To this end, we recall that for an arbitrary lattice $L$ and a set $X, L^{X}$ is also a lattice with the ordering defined pointwise and with properties analogous to those of the lattice $L$.

Proposition 3. Let $\mathcal{C}$ be the category of complete $\bigvee$-semilattices with complete semilattice homomorphisms. Then, there exists a morphism of powerset pairs

$$
(H, \Lambda, \Theta):\left(\operatorname{Set} \times \operatorname{Set}_{1} \times \mathcal{C}, \mathbf{T}\right) \rightarrow(\operatorname{Set} \times \mathcal{C}, \mathbf{W}),
$$

called a transformation of a fuzzy soft sets to fuzzy sets.

Proof. For arbitrary morphism $(f, g, \varphi):(X, U, L) \rightarrow$ $(Y, V, M)$, let the functor $H:$ Set $\times \operatorname{Set}_{1} \times \mathcal{C} \rightarrow \operatorname{Set} \times \mathcal{C}$ be defined by

$$
\begin{aligned}
& H(X, U, L)=\left(X, L^{U}\right), \\
& H(f, g, \varphi)=(f,(g, \varphi) \underset{W}{\vec{W}}):\left(X, L^{U}\right) \rightarrow\left(Y, M^{V}\right),
\end{aligned}
$$

where $(g, \varphi) \underset{W}{\rightarrow}$ is a morphism from the definition of the variablebasis powerset theory $\mathbf{W}$. For an arbitrary object $(X, U, L)$, we set

$$
\begin{aligned}
& \Lambda_{(X, U, L)}: T(X, U, L) \rightarrow W\left(X, L^{U}\right), \\
& (E, s) \in T(X, U, L), \quad \Lambda_{(X, U, L)}(E, s): X \rightarrow L^{U}, \\
& x \in X, u \in U, \\
& \Lambda_{(X, U, L)}(E, s)(x)(u)= \begin{cases}s(x)(u), & x \in E, \\
0_{L}, & \text { otherwise, }\end{cases} \\
& \Theta_{(X, U, L)}=1_{X}: X=R(X, U, L) \rightarrow X=V\left(X, L^{U}\right) .
\end{aligned}
$$

It can be easily proven that $\Lambda_{(X, U, L)}$ is a complete $\bigvee$-semilattice homomorphism, that is, $\Lambda_{(X, U, L)}\left(\bigvee_{i}\left(E_{i}, s_{i}\right)\right)=\bigvee_{i} \Lambda_{(X, U, L)}$ $\left(E_{i}, s_{i}\right)$ holds. We prove that the following diagram commutes:

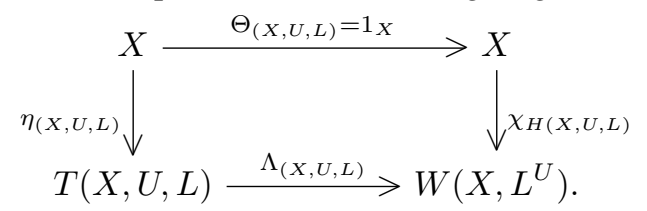


For $x, z \in X, u \in U$,

$$
\chi_{(H(X, U, L)}(x)(z)(u)= \begin{cases}1_{L}, & x=z \\ 0_{L}, & \text { otherwise. }\end{cases}
$$

Moreover,

$$
\begin{aligned}
& \Lambda_{(X, U, L)}\left(\eta_{(X, U, L)}(x)\right)=\Lambda_{(X, U, L)}\left(\{x\}, \eta_{x}\right): X \rightarrow L^{U}, \\
& \Lambda_{(X, U, L)}\left(\{x\}, \eta_{x}\right)(z)(u)= \begin{cases}\eta_{x}(x)(u)=1_{L}, & z=x, \\
0_{L}, & \text { otherwise },\end{cases}
\end{aligned}
$$

and the diagram commutes. Finally, we prove that for any morphism $(f, g, \varphi):(X, U, L) \rightarrow(Y, V, M)$, the following diagram commutes:

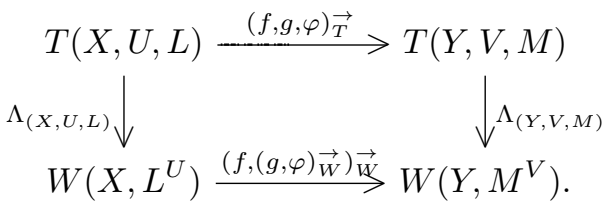

We recall that $g$ is a bijection map. For $(E, s) \in T(X, U, L)$ and $y \in Y, v \in V$, we have

$$
\begin{aligned}
& \left(f,(g, \varphi)_{W}^{\vec{W}}\right)_{W}^{\vec{W}}\left(\Lambda_{(X, U, L)}(E, s)\right)(y)(v) \\
& = \begin{cases}\bigvee_{x \in f^{-1}(y)}(g, \varphi)_{W}\left(\Lambda_{(X, U, L)}(E, s)(x)\right), & f^{-1}(y) \neq \emptyset, \\
0_{M}, & \text { otherwise }\end{cases} \\
& = \begin{cases}\bigvee_{x \in f^{-1}(y)} \varphi\left(\Lambda_{(X, U, L)}(E, s)(x)\left(g^{-1}(v)\right)\right), & f^{-1}(y) \neq \emptyset, \\
0_{M}, & \text { otherwise, }\end{cases} \\
& = \begin{cases}\bigvee_{x \in f^{-1}(y) \cap E} \varphi\left(s(x)\left(g^{-1}(v)\right)\right), & f^{-1}(y) \cap E \neq \emptyset, \\
0_{M}, & \text { otherwise }\end{cases}
\end{aligned}
$$

Moreover, we have

$$
\begin{aligned}
& \Lambda_{(X, U, L)}\left((f, g, \varphi)_{T}(E, s)(y)(v)\right) \\
& =\Lambda_{(X, U, L)}(f(E), s \rightarrow)(y)(v) \\
& = \begin{cases}s^{\rightarrow}(y)(v), & y \in f(E), \\
0_{M}, & \text { otherwise }\end{cases} \\
& = \begin{cases}\bigvee_{x \in f^{-1}(y) \cap E} \varphi\left(s(x)\left(g^{-1}(v)\right)\right), & f^{-1}(y) \cap E \neq \emptyset \\
0_{M}, & \text { otherwise. }\end{cases}
\end{aligned}
$$

Therefore, the diagram commutes, and $(H, \Lambda, \Theta)$ is a morphism of powerset pairs.
By a simple calculation we obtain the following corollary.

Corollary 1. The composition

$$
(H, \Lambda, \Theta) .(G, \Phi, \Psi):(\text { Set } \times \mathcal{C}, \mathbf{W}) \rightarrow(\text { Set } \times \mathcal{C}, W)
$$

is the identity morphism of powerset pairs.

\section{Fuzzy Soft Relations: An Application of Pow- erset Theory}

In set and fuzzy set theory, there are a number of concepts and methods that are, in fact, defined using powerset objects. To illustrate this, let us mention at least two typical powerset applications: relations and topology. If $\mathbf{T}=(T, \rightarrow, V, \eta)$ is an arbitrary powerset theory in a category $\mathbf{K}$, then a co-topology on an object $X$ of this category can be defined as the morphism $c$ : $T(X) \rightarrow T(X)$ in the category $C S L A T$ so that the following axioms are satisfied (see [25]):

1) $a \in T(X), \quad c(a) \geq a$,

2) $a, b \in T(X), \quad c(a \vee b)=c(a) \vee c(b)$,

3) $a \in T(X), \quad c(c(a))=c(a)$.

The set $\{a \in T(X): c(a)=a\}$ represents the set of all closed elements in $T(X)$. A morphism between co-topological spaces $(X, c)$ and $(Y, d)$ is a morphism $f: X \rightarrow Y$ in a category $\mathbf{K}$ such that $f_{T}: T(X) \rightarrow T(Y)$ is continuous, that is, for arbitrary $a \in T(X)$, we have $f_{T}(c(a)) \leq d\left(f_{T}(a)\right)$.

If, for example, instead of a category $\mathbf{K}$, we consider the category Set of sets and the powerset theory $\mathbf{P}$ or $\mathbf{Z}$ from examples 2 and 3 the obtained co-topology $c: P(X) \rightarrow P(X)$ represents the classical set topology in a set $X$, and $c: Z(X) \rightarrow$ $Z(X)$ represents a variant of a fuzzy topology, namely, the set of all closed elements of $Z(X)$ with respect to $c$ is the topology defined by C.L. Chang [26].

Herein, we focus on the relationship between powerset theory and relations, particularly between the fuzzy soft set powerset theory $\mathbf{T}$, or the monad $\widetilde{\mathbf{T}}$, which defines this powerset theory, and fuzzy soft relations in objects of the category Set $\times \operatorname{Set}_{0} \times$ C.

To clarify this relationship, we first consider the general situation of the powerset objects in a category that is defined by a monad in this category.

Hence, let $\mathbf{K}$ be a category, and let $\mathbf{T}=(T, \rightarrow, V, \chi)$ be a powerset theory in $\mathbf{K}$. Let $\mathbf{T}$ be defined by a monad $\widetilde{\mathbf{T}}=$ $(\widetilde{T}, \diamond, \eta)$ in $\mathbf{K}$. For a monad $\widetilde{\mathbf{T}}$, a new category $\mathbf{K}_{\widetilde{\mathbf{T}}}$ can be 
defined, called the Kleisli category of a monad $\widetilde{\mathbf{T}}$. We recall the definition of $\mathbf{K}_{\widetilde{\mathbf{T}}}$ (e.g., [19,27|).

Definition 6. Let $\mathbf{K}$ be a category, and let $\widetilde{\mathbf{T}}=(\widetilde{T}, \diamond, \eta)$ be a monad in $\mathbf{K}$. Then, the Kleisli category $\mathbf{K}_{\widetilde{\mathbf{T}}}$ of $\widetilde{\mathbf{T}}$ is defined as follows:

1) Objects of $\mathbf{K}_{\widetilde{\mathbf{T}}}$ are the same as objects of $\mathbf{K}$.

2) For arbitrary objects $A, B \in \mathbf{K}, \operatorname{Hom}_{\mathbf{K}_{\widetilde{\mathbf{T}}}}(A, B)=\operatorname{Hom}_{\mathbf{K}}$ $(A, \widetilde{T}(B))$. Morphisms in $\mathbf{K}_{\widetilde{\mathbf{T}}}$ are denoted by $A \rightsquigarrow B$.

3) A composition of morphisms $f: A \rightsquigarrow B, g: B \rightsquigarrow C$ is defined by $g \diamond f$.

If we understand the objects $\widetilde{T}(X)$ as an analog of the cloud of fuzzy objects over the object $X$, then the Klesli category in fact represents a category analogous to that of fuzzy morphisms between objects. Thus, this category is also used in areas other than the classical theory of fuzzy sets, for example, in computer science. This category also allows us to introduce the notion of a relation between objects of this category. Let us consider the following general definition.

Definition 7. Let $\mathbf{K}$ be a category, and let $\mathbf{T}=(T, \rightarrow, V, \chi)$ be a powerset theory in $\mathbf{K}$ defined by a monad $\widetilde{\mathbf{T}}=(\widetilde{T}, \diamond, \eta)$.

1) Let $X$ be an object of $\mathbf{K}$. Then, $R$ is a $T$-relation on $X$ if $R: V(X) \rightarrow T(X)$ is a morphism in Set such that $R=V(\widetilde{R})$, where $\widetilde{R}: X \rightarrow \widetilde{T}(X)$ is a morphism in $\mathbf{K}$.

2) Let $R$ and $S$ be $T$-relations on $X$ and $Y$, respectively. A morphism $f: R \rightarrow S$ between relations is a morphism $f: X \rightarrow Y$ in $\mathbf{K}$ such that in the diagram

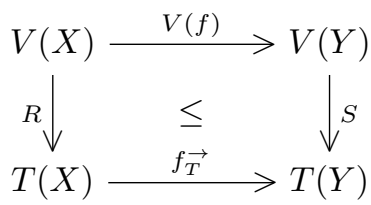

we have

$$
f_{T}^{\rightarrow} \cdot R \leq S . V(f)
$$

where $\leq$ is the pointwise ordering inherited from the semilattice $T(X)$.

This definition directly implies the possibility of composing two $T$-relations. In fact, let $R$ and $S$ be $T$-relations on $X$. Then, there exist morphisms $\widetilde{R}: X \rightarrow \widetilde{T}(X)$ and $\widetilde{S}: X \rightarrow$ $\widetilde{T}(X)$ such that $V(\widetilde{R})=R, V(\widetilde{S})=S$. The composition
$S \circ R: V(X) \rightarrow T(X)$ can be defined by the composition of morphisms $\widetilde{R}$ and $\widetilde{S}$ in the Kleisli category $\mathbf{K}_{\widetilde{\mathbf{T}}}$ :

$$
S \circ R:=V(\widetilde{S} \diamond \widetilde{R}): V(X) \rightarrow V \widetilde{T}(X)=T(X)
$$

The following examples illustrate the importance of $T$-relations in classical set and fuzzy set theories.

Example 7. Let $\mathbf{K}=$ Set, and let $\mathbf{P}$ be the classical powerset theory from Example 2 If $R$ is a $P$-relation $\mathrm{n} X$, then $R$ can be identified with the relation $R \subseteq X \times X$, and conversely, for any relation $R$ in $X, R$ is a $P$-relation. Morphisms $f: R \rightarrow S$ are maps $f: X \rightarrow Y$ such that

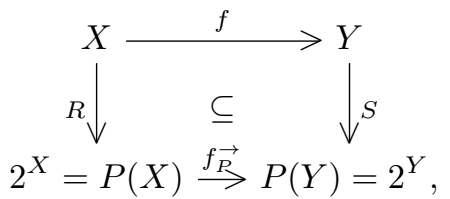

that is, $(x, y) \in R \Rightarrow(f(x), f(y)) \in S$ holds for arbitrary $x, y \in X$. As $\mathbf{P}$ is defined by the monad $\widetilde{\mathbf{P}}$ from Example 4 and any map $R: X \rightarrow P(X)$ is also a morphism $X \rightarrow \widetilde{P}(X)$, using $\diamond$, it is possible to define the composition of $P$-relations, and this composition is identical to the standard composition of relations in sets.

Therefore, the category of $P$-relations is isomorphic to the classical category of sets with relations with the corresponding morphisms. This category is isomorphic to the Kleisli category $\operatorname{Set}_{\widetilde{\mathbf{P}}}$.

Example 8. Let $\mathbf{K}=$ Set, and let $\mathbf{Z}=(Z, \rightarrow, V, \chi)$ be the classical Zadeh's powerset theory from Example 3. If $R$ is a $Z$-relation on $X, R$ can be identified with a fuzzy relation $R \curvearrowleft X \times X$, and conversely, any fuzzy relation $R: X \times X \rightarrow$ $L$ defines a $Z$-relation. Morphisms $f: R \rightarrow S$ are maps $f: X \rightarrow Y$ such that

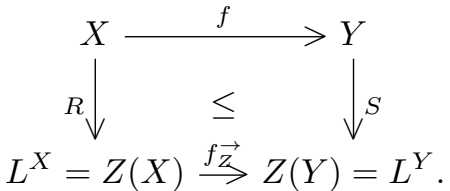

It can be easily proven that $f$ is a morphism if and only if

$$
R\left(x, x^{\prime}\right) \leq S\left(f(x), f\left(x^{\prime}\right)\right)
$$

for each $x, x^{\prime} \in X$. As $\mathbf{Z}$ is defined by the monad $\widetilde{\mathbf{Z}}$ from Example 5, and any map $R: X \rightarrow Z(X)$ is also a morphism $X \rightarrow \widetilde{Z}(X)$, using $\diamond$, it is possible to define the composition of $Z$-relations, and this composition is identical to the standard 
composition of $L$-valued fuzzy relations. Hence, the category of $Z$-relational pairs is isomorphic to the standard category of $L$-fuzzy relations.

Both Examples 7 and 8 indicate that the classical relations in sets or fuzzy relations can be fully replaced by morphisms in Kleisli categories. This now provides the opportunity to consider fuzzy soft relations defined on the basic objects of fuzzy soft sets from the perspective of Kleisli categories. Although it is common practice in fuzzy set theory to explicitly define new terms without justifying motivation, it is always advisable to attempt to interpret such an established concept in the context of a broader theory. An example of this procedure can be the aforementioned notion of relation in fuzzy structures. Using the standard procedure, we can independently define a fuzzy soft relation in a space $(X, U, L)$, even in various ways. However, if we realize the previous relationship between relations in sets and the monad $\mathbf{P}$, or between fuzzy relations in fuzzy sets and the monad $\mathbf{Z}$, then the correct procedure requires that the exclusive definition of a fuzzy soft relation also follows an analogous relationship between fuzzy soft relations and the Kleisli category $\mathbf{K}_{\widetilde{\mathbf{T}}}$.

If we use the common procedure in the theory of fuzzy sets, we can introduce, for example, the following explicit definition of a fuzzy soft relation in a space $(X, U, L)$, a composition of such fuzzy soft relations and morphisms between fuzzy soft relations in different spaces.

Definition 8. Let $(X, U, L)$ be an object in $\mathbf{K}=$ Set $\times$ $\operatorname{Set}_{1} \times \mathcal{C}$.

1) A fuzzy soft relation in $(X, U, L)$ is a fuzzy soft set in the space $(X \times X, U, L)$.

2) If $(E, R)$ and $(F, S)$ are fuzzy soft relations in $(X, U, L)$, their composition is a fuzzy soft relation defined by

$$
\begin{aligned}
& (F, S) \oplus(E, R)=(E . F, S \circ R), \\
& E . F=\left\{(x, z) \in X^{2}: \exists y \in X,(x, y) \in E,(y, z) \in F\right\}, \\
& (x, z) \in E . F, \\
& (S \circ R)(x, z)(u) \\
& =\bigvee_{\{y \mid(x, y) \in E,(y, z) \in F\}} R(x, y)(u) \otimes S(y, z)(u) .
\end{aligned}
$$

3) Let $(E, R)$ and $(F, S)$ be fuzzy soft relations in $(X, U, L)$ and $(Y, V, M)$, respectively. Then, $(f, g, \varphi):(E, R) \rightarrow$ $(F, S)$ is a morphism of fuzzy soft relations if (a) $(f, g, \varphi):(X, U, L) \rightarrow(Y, V, M)$ is a morphism in the category $\mathbf{K}$.

(b) For arbitrary $(a, b) \in E$ and $u \in U$, we have $(f(a), f(b))$ $\in F$ and

$$
\varphi(R(a, b)(u)) \leq S(f(a), f(b))(g(u))
$$

However, to maintain the analogy of the relationships between sets, fuzzy sets, and fuzzy soft sets, we should show that, as in the case of relations in sets or fuzzy relations in fuzzy sets, fuzzy soft relations can be derived from morphisms in the Kleisli category of the monad $\widetilde{\mathbf{T}}$ defining the fuzzy soft set powerset theory $\mathbf{T}$, and that the composition of fuzzy soft relations corresponds to the composition of morphisms in the Kleisli category. We prove this in the next proposition.

Proposition 4. Let $\mathbf{T}=(T, \rightarrow, R, \eta)$ be the fuzzy soft set powerset theory from Theorem 2 , and let $\widetilde{\mathbf{T}}=(\widetilde{T}, \odot, \xi)$ be the monad from Theorem 3 in the category $\mathbf{K}=\mathbf{S e t} \times \mathbf{S e t}_{1} \times \mathcal{C}$ defining $\mathbf{T}$. For an object $\mathcal{X}=(X, U, L)$ of this category, we set

$$
\begin{aligned}
\mathcal{R}(\mathcal{X})= & \{(E, S) \mid(E, S) \\
\text { is a fuzzy soft relation (Definition 8) in } \mathcal{X}\}, & \\
\mathcal{R}_{T}(\mathcal{X})= & \{r \mid r: X \rightarrow T(\mathcal{X}) \\
& \text { is a } T \text {-relation (Definition 7) on } \mathcal{X}\} .
\end{aligned}
$$

1) There exists a bijective map

$$
\alpha: \mathcal{R}(\mathcal{X}) \stackrel{\cong}{\lessgtr} \mathcal{R}_{T}(\mathcal{X}) .
$$

2) If $(E, Q),(F, S) \in \mathcal{R}(\mathcal{X})$, then

$$
\alpha((F, S) \oplus(E, Q))=\alpha(F, S) \diamond \alpha(E, Q),
$$

where the clone composition $\diamond$ is defined by relations (3) and (4).

Proof. 1) Let $(E, S)$ be a fuzzy soft relation in $\mathcal{X}$ defined in Definition 8. To define the map $\alpha$, for arbitrary $x \in X$, we set $X_{x}^{E}=\{z \in X:(z, x) \in E\}$. Clearly, $E=\bigcup_{x \in X} X_{x}^{E} \times\{x\}$, and we can define a map $\alpha$ by

$$
\begin{aligned}
& \alpha(E, R)=r: X \rightarrow T(\mathcal{X}), \\
& \forall x \in X, \quad r(x)=\left(X_{x}^{E}, S_{x}\right) \in T(\mathcal{X}), \\
& z \in X_{x}^{E}, u \in U, \quad S_{x}(z)(u):=S(z, x)(u) .
\end{aligned}
$$

Conversely, let $s: X \rightarrow T(\mathcal{X})$ be a map, where, using the 
notation (2), we have

$$
\forall x \in X, \quad s(x)=\left(X_{x}^{s}, s_{x}\right) \in T(\mathcal{X})
$$

$X_{x}^{s} \subseteq X, s_{x}: X_{x}^{s} \rightarrow L^{U}$. We define a fuzzy soft relation $\alpha^{-1}(s)=(F, Q)$ in $\mathcal{X}$ by

$$
\begin{aligned}
& F=\bigcup_{x \in X} X_{x}^{s} \times\{x\} \subseteq X \times X, \\
& (y, x) \in F, u \in U, \quad Q(y, x)(u)=s_{x}(y)(u) .
\end{aligned}
$$

By a simple calculation, we obtain $\alpha^{-1} \cdot \alpha(E, S)=(E, S)$ and $\alpha . \alpha^{-1}(s)=s$.

2) Let $(E, Q),(F, S)$ be fuzzy soft relations in $(X, U, L)$, and let

$$
\begin{aligned}
& \alpha(E, Q)=r, \quad \alpha(F, S)=s, \\
& \forall x \in X, \quad r(x)=\left(X_{x}^{E}, r_{x}\right), \quad s(x)=\left(X_{x}^{F}, s_{x}\right) .
\end{aligned}
$$

According to identities (3), (4), and (5), we obtain

$$
\begin{aligned}
& x \in X, \quad(s \diamond r)(x)=\left(X_{x}^{s \diamond r},(s \diamond r)_{x}\right), \\
& X_{x}^{s \diamond r}=\bigcup_{y \in X_{x}^{E}} X_{y}^{F}, \\
& z \in X_{x}^{s \diamond r}, \\
& (s \diamond r)_{x}(z)=\bigvee_{\left\{y \in X_{x}^{E} \mid z \in X_{y}^{F}\right\}} r_{x}(y)(u) \otimes s_{y}(z)(u) \\
& =\bigvee_{\{y \mid(x, y) \in E,(y, z) \in F\}} Q(x, y)(u) \otimes S(y, z)(u) \\
& =S \circ Q(x, z) .
\end{aligned}
$$

It follows that $\bigcup_{x \in X} X_{x}^{s \diamond r} \times\{x\}=E . F$ and $s \diamond r=\alpha(E . F, S \circ$ $Q)=\alpha((F, S) \oplus(E, Q))$.

\section{Conclusion}

In this study, we focused on constructing a fuzzy soft set powerset theory, including its variable-basis variant, that enables the use of $L$-valued fuzzy soft sets for various lattices $L$. We proved that there exists a variable-basis fuzzy soft set powerset theory in the category Set $\times \operatorname{Set}_{0} \times \mathcal{C}$, which is a generalization of both the classical and fuzzy set powerset theory in the category Set and the variable-basis fuzzy set powerset theory in the category Set $\times \mathcal{C}$. We proved that this powerset theory is also defined by a suitable monad in the category Set $\times \operatorname{Set}_{1} \times \mathcal{C}$. This allows us to use a series of constructs from the theory of fuzzy sets that, by their very nature, require the existence of a powerset theory defined by a monad. As an example, we used the monad defining a fuzzy soft set powerset theory to define fuzzy soft relations and compositions of these relations.

\section{Conflict of Interest}

No potential conflict of interest relevant to this article was reported.

\section{Acknowledgement}

This research was partially supported by the ERDF/ESF project CZ.02.1.01/0.0/0.0/17-049/0008414.

\section{References}

[1] D. Molodtsov, "Soft set theory-first results," Computers \& Mathematics with Applications, vol. 37. no. 4-5, pp. 1931, 1999. https://doi.org/10.1016/S0898-1221(99)000565

[2] P. K. Maji, R. Biswas, and A. R. Roy, "Soft set theory," Computers \& Mathematics with Applications, vol. 45, no. 4-5, pp. 555-562, 2003. https://doi.org/10.1016/S08981221(03)00016-6

[3] P. K. Maji, A. R. Roy, and R. Biswas, "An application of soft sets in a decision making problem," Computers \& Mathematics with Applications, vol. 44, no. 8-9, pp. 1077-1083, 2002. https://doi.org/10.1016/s0898-1221(02) 00216-X

[4] P. Majumdar and S. K. Samanta, "Similarity measure of soft sets," New Mathematics and Natural Computation, vol. 4, no. 1, pp. 1-12, 2008. https://doi.org/10.1142/ S1793005708000908

[5] H. Aktas and N. Cagman, "Soft sets and soft groups," Information Sciences, vol. 177, no. 13, pp. 2726-2735, 2007. https://doi.org/10.1016/j.ins.2006.12.008

[6] F. Feng, Y. B. Jun, and X. Zhao, "Soft semirings," Computers \& Mathematics with Applications, vol. 56, no. 10, pp. 2621-2628, 2008. https://doi.org/10.1016/j.camwa.2008. 05.011

[7] D. Chen, E. C. C. Tsang, D. S. Yeung, and X. Wang, "The parameterization reduction of soft sets and its applications," Computers \& Mathematics with Applications, vol. 
49, no. 5-6, pp. 757-763, 2005. https://doi.org/10.1016/j. camwa.2004.10.036

[8] M. M. Mushrif, S. Sengupta, and A. K. Ray, "Texture classification using a novel, soft-set theory based classification algorithm," in Computer Vision - ACCV2006. Heidelberg, Germany: Springer, 2006, pp. 246-254. https: //doi.org/10.1007/11612032_26

[9] P. K. Maji, A. R. Roy, and R. Biswas, "Fuzzy soft sets," Journal of Fuzzy Mathematics, vol. 9, no. 3, pp. 589-602, 2001.

[10] B. Tanay and M. B. Kandemir, "Results on fuzzy soft functions," New Trends in Mathematical Sciences, vol. 3, no. 4, pp. 1-17, 2015.

[11] K. Athar and B. Ahmad, "Mappings on fuzzy soft classes," Advances in Fuzzy Systems, vol. 2009, article no. 407890, 2009. https://doi.org/10.1155/2009/407890

[12] A. R. Roy and P. K. Maji, "A fuzzy soft set theoretic approach to decision making problems," Journal of Computational and Applied Mathematics, vol. 203, no. 2, pp. 412-418, 2007. https://doi.org/10.1016/j.cam.2006.04.008

[13] Z. Xiao, K. Gong, and Y. Zou, "A combined forecasting approach based on fuzzy soft sets," Journal of Computational and Applied Mathematics, vol. 228, no. 1, pp. 326-333, 2009. https://doi.org/10.1016/j.cam.2008.09.033

[14] Y. Zou and Z. Xiao, "Data analysis approaches of soft sets under incomplete information," Knowledge-Based Systems, vol. 21, no. 8, pp. 941-945, 2008. https://doi.org/ 10.1016/j.knosys.2008.04.004

[15] D. Pei and D. Miao, "From soft sets to information systems," in Proceedings of 2005 IEEE International Conference on Granular Computing, Beijing, China, 2005, pp. 617-621. https://doi.org/10.1109/GRC.2005.1547365

[16] D. V. Kovkov, V. M. Kolbanov, and D. A. Molodtsov, "Soft sets theory-based optimization," Journal of Computer and Systems Sciences International, vol. 46, no. 6, pp. 872880, 2007. https://doi.org/10.1134/S1064230707060032

[17] L. A. Zadeh, "Fuzzy sets," Information and Control, vol. 8, no. 3, pp. 338-353, 1965. https://doi.org/10.1016/S00199958(65)90241-X
[18] S. E. Rodabaugh, "Powerset operator foundations for poslat fuzzy set theories and topologies," in Mathematics of Fuzzy Sets. Boston, MA: Springer, 1999, pp. 91-116. https://doi.org/10.1007/978-1-4615-5079-2_3

[19] E. G. Manes, Algebraic Theories. Berlin, Germany: Springer-Verlag, 1976.

[20] S. E. Rodabaugh, "Relationship of algebraic theories to powerset theories and fuzzy topological theories for lattice-valued mathematics," International Journal of Mathematics and Mathematical Sciences, vol. 2007, article no. 043645, 2007. https://doi.org/10.1155/2007/43645

[21] V. Novak, I. Perfilijeva, and J. Mockor, Mathematical Principles of Fuzzy Logic. Boston, MA: Kluwer Academic Publishers, 1999.

[22] J. Mockor, and P. Hurtik, "Fuzzy soft sets and image processing application," in 14th International Conference on Theory and Application of Fuzzy Systems and Soft Computing - ICAFS-2020. Singapore: Springer International Publishing, 2020. https://doi.org/10.1007/978-3-030-64058-3

[23] S. Mac Lane, Categories for the Working Mathematician, 2nd ed. New York, NY: Springer, 1998.

[24] S. E. Rodabaugh, "Powerset operator based foundation for point-set lattice-theoretic (poslat) fuzzy set theories and topologies," Quaestiones Mathematicae, vol. 20, no. 3, pp. 463-530, 1997. https://doi.org/10.1080/16073606. 1997.9632018

[25] J. A. Goguen, "The fuzzy Tychonoff theorem," Journal of Mathematical Analysis and Applications, vol. 43, no. 3, pp. 734-742, 1973. https://doi.org/10.1016/0022247X(73)90288-6

[26] C. L. Chang, "Fuzzy topological spaces," Journal of Mathematical Analysis and Applications, vol. 24, no. 1, pp. 182190, 1968. https://doi.org/10.1016/0022-247x(68)90057-7

[27] H. Kleisli, "Every standard construction is induced by a pair of adjoint functors," Proceedings of the American Mathematical Society, vol. 16, no. 3, pp. 544-546, 1965. https://doi.org/10.2307/2034693

[28] J. Mockor, "Powerset operators of extensional fuzzy sets," Iranian Journal of Fuzzy Systems, vol. 15, no. 2, pp. 143163, 2008. https://dx.doi.org/10.22111/ijfs.2018.3765 
[29] J. Mockor, "Cut systems in sets with similarity relations," Fuzzy Sets and Systems, vol. 161, no. 24, pp. 3127-3140, 2010. https://doi.org/10.1016/j.fss.2010.07.009

[30] J. Mockor and M. Holcapek, "Fuzzy objects in spaces with fuzzy partitions," Soft Computing, vol. 21, no. 24, pp. 7269-7284, 2017. https://doi.org/10.1007/s00500-0162431-4

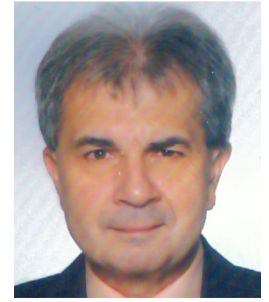

Jiří Močkoř is a Professor of algebra at the University of Ostrava and Senior Researcher at the Institute for Research and Application of Fuzzy Modeling at this University. He has been working on research related to category theory with applications in fuzzy set theory and fuzzy logic. He is the author or co-author of 4 monographs on theoretical algebra and fuzzy logic and published more than 100 articles in peer-reviewed journals and conferences.

E-mail: mockor@osu.cz 